\title{
Anatomy of geodesic Witten diagrams
}

\author{
Heng-Yu Chen, ${ }^{a}$ En-Jui Kuo ${ }^{a}$ and Hideki Kyono ${ }^{b}$ \\ ${ }^{a}$ Department of Physics and Center for Theoretical Sciences, National Taiwan University, \\ Taipei 10617, Taiwan \\ ${ }^{b}$ Department of Physics, Kyoto University, \\ Kitashirakawa Oiwake-cho, Kyoto 606-8502, Japan \\ E-mail: heng.yu.chen@phys.ntu.edu.tw, r04222087@ntu.edu.tw, \\ h_kyono@gauge.scphys.kyoto-u.ac.jp
}

ABSTRACT: We revisit the so-called "Geodesic Witten Diagrams" (GWDs) [1], proposed to be the holographic dual configuration of scalar conformal partial waves, from the perspectives of CFT operator product expansions. To this end, we explicitly consider three point GWDs which are natural building blocks of all possible four point GWDs, discuss their gluing procedure through integration over spectral parameter, and this leads us to a direct identification with the integral representation of CFT conformal partial waves. As a main application of this general construction, we consider the holographic dual of the conformal partial waves for external primary operators with spins. Moreover, we consider the closely related "split representation" for the bulk to bulk spinning propagator, to demonstrate how ordinary scalar Witten diagram with arbitrary spin exchange, can be systematically decomposed into scalar GWDs. We also discuss how to generalize to spinning cases.

Keywords: AdS-CFT Correspondence, Conformal Field Theory

ARXIV EPRINT: 1702.08818 


\section{Contents}

1 Introduction 1

2 Scalar four point geodesic Witten diagrams revisited 3

3 Spinning three point functions and conformal blocks 11

4 Spinning conformal partial waves from Anti-de Sitter space 14

$\begin{array}{ll}4.1 \text { The }\left(l_{1}, l_{2}, 0\right) \text { case } & 17\end{array}$

$\begin{array}{lll}4.2 & \text { The }(1,1,2) \text { case } & 18\end{array}$

5 Decomposition of Witten diagrams via split representation 20

$\begin{array}{lr}\text { A Embedding formalism } & 29\end{array}$

B Integrals for three point geodesic Witten diagrams $\quad 32$

C Integrals for three point normal Witten diagrams 33

D Rewriting tensor structures and some useful identities $\quad 34$

E Computational details for decomposition analysis 36

\section{Introduction}

One of the most powerful applications of AdS/CFT correspondence is that we can realize the important and sometimes complicated CFT observables such as correlation functions, through computationally simple geometric configurations inside the dual Anti-de Sitter space. (See [2, 3] for selected references, and [5] for a good review in this area.) Such an application often relies heavily on the underlying conformal symmetries or equivalently the isometries of Anti-de Sitter space. Conformal blocks, which allow us to disentangle what are universally constrained by conformal symmetries in four point CFT correlation functions, from theory-dependent data, such as spectrum of scale dimensions $\left\{\Delta_{i}\right\}$ and OPE coefficients $\left\{\lambda_{i j k}\right\}$, offer a ideal venue for such a geometric realization in the dual AdS space.

Curiously, despite almost twenty years since the inception of AdS/CFT correspondence, the holographic dual configuration of conformal block, termed "geodesic Witten diagram" (GWD), have only been constructed recently in a striking paper [1]. In a complete analogy with the CFT decomposition, the ordinary scalar four point Witten diagrams which holographically computes the full four point CFT correlation functions, can be shown to decompose into a summation over the GWDs. Moreover, each of these scalar GWDs 
involved in the sum, can be identified directly with the conformal block for single and double trace primary operator exchange.

However from the perspective of CFT operator product expansions, it is sometimes more illuminating to think instead about the individual conformal block $G_{\Delta, J}(u, v)$ as being built from fusing a pair of three point functions, each involving two of the external primary operators and the internal exchange operator $\mathcal{O}_{\Delta, J}$ itself (for good recent CFT reviews, see $[6,7])$. Indeed this fusion procedure of three point functions was made explicit in [8] (and later extended in [9]), through defining the so-called "shadow operators", which yields the integral representation of conformal block. This will be reviewed in the next section. It is therefore natural to ask if this further decomposition procedure of individual conformal blocks themselves can also be seen in AdS space, perhaps directly cutting up a four point GWD in the middle into two three point ones? It turns out that this intuitive picture is qualitatively correct, and the detailed justification comes from the non-trivial identity between the bulk to bulk and bulk to boundary propagators we shall derive. We shall name the resulting building element: three point geodesic Witten diagram, see figure 3 . The main difference from the ordinary three point Witten diagram is now that the bulk interaction point is restricted to move along the geodesic connecting two our of three boundary points. As we will see in section 2, this procedure of cutting and rejoining also allows us to directly identify four point scalar GWD with the integral representation of scalar conformal block by construction, hence provides an alternative proof for the results in [1].

As an main application of this understanding, the three point GWDs become particularly useful when constructing the holographic dual of spinning conformal blocks [10, 11], as they allow us to directly apply the earlier general parameterization of three point vertex for three symmetric traceless tensor fields constructed in $[12,13]$ (up to certain modification to account for the restriction along the geodesic) to study the precise nature of the interaction. The resultant calculations can then be expressed in terms of appropriate CFT tensor structures, we will provide explicit examples and illustrate how general spinning geodesic Witten diagrams can be constructed in section 4 . We will review the relevant CFT details in section 3 .

Finally, the analysis we have done is closely related to the so-called "Split representation" of bulk to bulk propagator $[14,15]$. in fact we will demonstrate its power by combining with the knowledge of three point GWDs in section 5. Explicitly we can rewrite the split representation of four point scalar Witten diagrams with arbitrary spin- $J$ exchange into a summation over products of three point GWDs. By explicitly identifying the physical residues when performing the integration over so-called "spectral parameter" $\nu$, we can show that the summation contains one four point scalar GWD for single trace operator with spin- $J$, plus infinite towers of four point scalar GWDs for double trace primary operators with spins $0,1, \ldots, J$. This is consistent with and completes analysis in [1] for $J=0,1$ cases. We also discuss how similar decompositions can be done for spinning Witten diagrams into spinning GWDs.

We relegate some useful background materials and computational details into several appendices. 
While this work is being finalized, two nice preprints $[16,17]$ appeared,$^{1}$ which have partial overlaps with our results. However we hope our independent work, which has somewhat different computational approaches and topical emphases, can complement their works. An earlier work [18], which considered holographic dual of conformal block with single external operator with spin, also contained a special case of our results. ${ }^{2}$

\section{Scalar four point geodesic Witten diagrams revisited}

Let us begin by reviewing the essential details about the geodesic Witten diagram in $d+1$ dimensional Anti-de Sitter space $\mathrm{AdS}_{d+1}$ [1]. This was proposed to be the holographic dual configuration of the $d$-dimensional scalar conformal partial wave associated with the exchange of a primary operator $\mathcal{O}_{\Delta, J}$ of scaling dimension $\Delta$ and spin $J$ and its conformal descendants between two pairs of external local scalar primary operators $\mathcal{O}_{\Delta_{1}}\left(P_{1}\right), \mathcal{O}_{\Delta_{2}}\left(P_{2}\right)$ and $\mathcal{O}_{\Delta_{3}}\left(P_{3}\right), \mathcal{O}_{\Delta_{4}}\left(P_{4}\right)$ :

$$
W_{\mathcal{O}_{\Delta, J}}\left(P_{1}, P_{2}, P_{3}, P_{4}\right)=\left(\frac{P_{24}}{P_{14}}\right)^{\frac{\Delta_{12}}{2}}\left(\frac{P_{14}}{P_{13}}\right)^{\frac{\Delta_{34}}{2}} \frac{G_{\mathcal{O}_{\Delta, J}}(u, v)}{\left(P_{12}\right)^{\frac{\Delta_{1}+\Delta_{2}}{2}}\left(P_{34}\right)^{\frac{\Delta_{3}+\Delta_{4}}{2}}},
$$

where $\Delta_{i j}=\Delta_{i}-\Delta_{j}$. In this note we will mostly use so-called "embedding formalism" reviewed in appendix A and follow the conventions in [6]. Here $P_{i}$ labels the position of operator $\mathcal{O}_{\Delta_{i}}\left(P_{i}\right)$ in $d+2$ dimensional embedding space, and their separations are:

$$
P_{i j}=-2 P_{i} \cdot P_{j}, \quad i, j=1,2,3,4 .
$$

We can express the "scalar conformal block" $G_{\mathcal{O}_{\Delta, J}}(u, v)$ for $\mathcal{O}_{\Delta, J}$ as a function of the two independent conformally invariant cross-ratios:

$$
u=\frac{P_{12} P_{34}}{P_{13} P_{24}}, \quad v=\frac{P_{14} P_{23}}{P_{13} P_{24}} .
$$

The closed form expressions of $G_{\mathcal{O}_{\Delta, J}}(u, v)$ for even $d$-dimensions have been solved explicitly in terms of hypergeometric functions using quadratic Casimir operators [8, 20]; more recently the precise connections of $G_{\mathcal{O}_{\Delta, J}}(u, v)$ with the eigenfunctions of quantum integrable systems have also been established for arbitrary $d$-dimensions in [21, 22].

Now imagine these external scalar primary operators $\left\{\mathcal{O}_{\Delta_{i}}\right\}$ are inserted at the boundary of $\operatorname{AdS}_{d+1}$ at points $\left\{P_{i}\right\}$, and use $\gamma_{12}$ and $\gamma_{34}$ to denote the geodesics connecting the points $P_{1,2}$ and $P_{3,4}$ respectively, the four point scalar geodesic Witten diagram is defined through the double integral (see figure 1):

$$
\begin{aligned}
& \mathcal{W}_{\Delta, J}\left(P_{i}\right)= \\
& \int_{\gamma_{12}} \int_{\gamma_{34}} \prod_{c=1}^{2} \Pi_{\Delta_{c}}\left(X(\lambda), P_{c}\right) \hat{\Pi}_{\Delta, J}\left(X(\lambda), \tilde{X}\left(\lambda^{\prime}\right) ; \frac{d X(\lambda)}{d \lambda}, \frac{d \tilde{X}\left(\lambda^{\prime}\right)}{d \lambda^{\prime}}\right) \prod_{c^{\prime}=3}^{4} \Pi_{\Delta_{c^{\prime}}}\left(\tilde{X}\left(\lambda^{\prime}\right), P_{c^{\prime}}\right) .
\end{aligned}
$$

\footnotetext{
${ }^{1}$ Another nice paper [4] has appeared simultaneously when we submitted version 1 of this work to arXiv, and their work also has some partial overlaps.

${ }^{2}$ Please also see [19] for the interesting connections between so-called "OPE blocks" and geodesic Witten diagrams.
} 


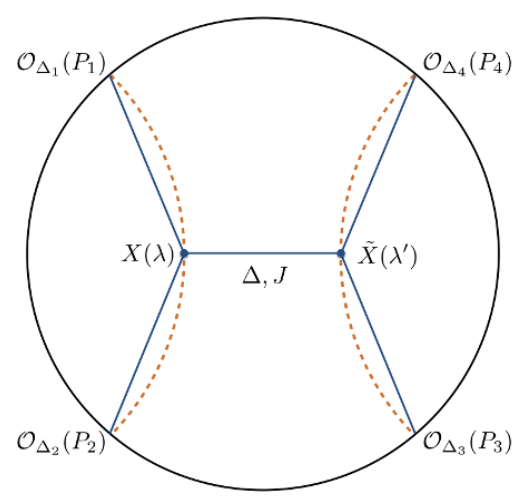

Figure 1. Four point scalar geodesic Witten diagram where the orange curves describe the geodesics and the blue lines are bulk to boundary propagators, such that the interaction vertices move along geodesics $\gamma_{12}$ and $\gamma_{34}$.

Here $-\infty<\lambda, \lambda^{\prime}<+\infty$ are the line parameters of $\gamma_{12}$ and $\gamma_{34}$ which we integrate along with, in terms of bulk $\operatorname{AdS}_{d+1}$ coordinates $X^{A}(\lambda)$ and $\tilde{X}^{A}\left(\lambda^{\prime}\right)$, the two geodesics are given by following curves:

$$
\gamma_{12}: X^{A}(\lambda)=\frac{P_{1}^{A} e^{\lambda}+P_{2}^{A} e^{-\lambda}}{\left(P_{12}\right)^{\frac{1}{2}}}, \quad \gamma_{34}: \tilde{X}^{A}\left(\lambda^{\prime}\right)=\frac{P_{3}^{A} e^{\lambda^{\prime}}+P_{4}^{A} e^{-\lambda^{\prime}}}{\left(P_{34}\right)^{\frac{1}{2}}} .
$$

The integrand in (2.4) consists of the pull-back of bulk to boundary scalar propagators: ${ }^{3}$

$$
\Pi_{\Delta_{1,2}}\left(X(\lambda), P_{1,2}\right)=\frac{\mathcal{C}_{\Delta_{1,2}}}{\left(-2 P_{1,2} \cdot X(\lambda)\right)^{\Delta_{1,2}}}, \quad \Pi_{\Delta_{3,4}}\left(\tilde{X}\left(\lambda^{\prime}\right), P_{3,4}\right)=\frac{\mathcal{C}_{\Delta_{3,4}}}{\left(-2 P_{3,4} \cdot \tilde{X}\left(\lambda^{\prime}\right)\right)^{\Delta_{3,4}}},
$$

and the pull-back of bulk to bulk propagator of spin- $J$ tensor field between $X^{A}(\lambda)$ and $\tilde{X}^{A}\left(\lambda^{\prime}\right)$ on the two geodesics:

$$
\begin{aligned}
& \hat{\Pi}_{\Delta, J}\left(X(\lambda), \tilde{X}\left(\lambda^{\prime}\right) ; \frac{d X(\lambda)}{d \lambda}, \frac{d \tilde{X}\left(\lambda^{\prime}\right)}{d \lambda^{\prime}}\right) \\
& =\left(\frac{d X(\lambda)}{d \lambda}\right)_{A_{1}, \ldots A_{J}}\left(\frac{d \tilde{X}\left(\lambda^{\prime}\right)}{d \lambda^{\prime}}\right)_{\tilde{A}_{1}, \ldots, \tilde{A}_{J}} \Pi_{\Delta, J}^{\left\{A_{1} \ldots A_{J}\right\},\left\{\tilde{A}_{1} \ldots \tilde{A}_{J}\right\}}(X, \tilde{X}) .
\end{aligned}
$$

Here $\Pi_{\Delta, J}^{\left\{A_{1} \ldots A_{J}\right\},\left\{\tilde{A}_{1} \ldots \tilde{A}_{J}\right\}}(X, \tilde{X})$ is a (doubly) symmetric, traceless and transverse (STT) tensor whose form will be specified momentarily, such that each set of indices satisfy $X_{A_{1}} \Pi^{\left\{A_{1} A_{2} \ldots A_{J}\right\}}=0$ and $\eta_{A_{1} A_{2}} \Pi^{\left\{A_{1}, A_{2} \ldots A_{J}\right\}}=0$. In (2.7), we have introduce the shorthand notation:

$$
Y^{A_{1} \ldots A_{J}} \equiv Y^{A_{1}} \ldots Y^{A_{J}}
$$

to denote symmetric tensor built from the products of identical vector or vectorial operator $Y^{A}$. The proposal of geodesic Witten diagram [1] is such that instead of integrating the

\footnotetext{
${ }^{3}$ Here the overall normalization constant $\mathcal{C}_{\Delta}=\frac{\Gamma(\Delta)}{2 \pi^{\frac{d}{2}} \Gamma\left(\Delta+1-\frac{d}{2}\right)}$ is defined as a special case of (2.18).
} 
bulk interacting vertices $(X, \tilde{X})$ over the entire $\operatorname{AdS}_{d+1}$ as in computing the holographic correlation functions, they are pulled back to move only along the geodesic trajectories (2.5) and the integration in (2.4) is taken along the line parameters $\lambda$ and $\lambda^{\prime}$. By showing (2.4) satisfies the eigenvalue equation of quadratic conformal Casimir operator, the authors of [1] explicitly established:

$$
\mathcal{W}_{\Delta, J}\left(P_{i}\right) \equiv W_{\mathcal{O}_{\Delta, J}}\left(P_{i}\right)
$$

up to an unimportant overall normalization constant, in our subsequent computations, we will do the same unless otherwise stated. Moreover, we will provide an alternative proof for (2.9) by considering three point geodesic Witten diagrams momentarily.

The doubly STT tensor $\Pi_{\Delta, J}^{\left\{A_{1} \ldots A_{J}\right\},\left\{\tilde{A}_{1} \ldots \tilde{A}_{J}\right\}}(X, \tilde{X})$ in (2.7) can be obtained from the following index-free generating polynomial [14]:

$$
\Pi_{\Delta, J}(X, \tilde{X} ; W, \tilde{W})=\sum_{k=0}^{J}(W \cdot \tilde{W})^{J-k}((W \cdot \tilde{X})(\tilde{W} \cdot X))^{k} g_{k}(\mathrm{u}), \quad \mathrm{u}=-1-X \cdot \tilde{X}
$$

where $W^{A}$ (and $\tilde{W}^{A}$ ) is the auxiliary polarization vector satisfying $W \cdot X=W \cdot W=0$ and the function $g_{k}(\mathrm{u})$ can be explicitly obtained from the equation of motion for a massive spin $J$ particle in terms of hypergeometric functions. Next we act on (2.10) with the product of projection operators $K_{A}$ and $\tilde{K}_{\tilde{A}}$ :

$$
\begin{aligned}
& \Pi_{\Delta, J}^{\left\{A_{1} \ldots A_{J}\right\},\left\{\tilde{A}_{1} \ldots \tilde{A}_{J}\right\}}(X, \tilde{X})=\frac{1}{\left[J !\left(\frac{d-1}{2}\right)_{J}\right]^{2}} K^{A_{1} \ldots A_{J}} \tilde{K}^{\tilde{A}_{1} \ldots \tilde{A}_{J}} \Pi_{\Delta, J}(X, \tilde{X} ; W, \tilde{W}) \\
& =\sum_{k=0}^{J} G_{B_{1}}^{\left\{A_{1}\right.} \ldots G_{B_{J}}^{\left.A_{J}\right\}} \tilde{G}_{\tilde{B}_{1}}^{\left\{\tilde{A}_{1}\right.} \ldots \tilde{G}_{\tilde{B}_{J}}^{\left.\tilde{A}_{J}\right\}} \eta^{B_{1} \tilde{B}_{1}} \ldots \eta^{B_{J-k} \tilde{B}_{J-k}} \tilde{X}^{B_{J-k+1}} \ldots \tilde{X}^{B_{J}} X^{\tilde{B}_{J-k+1}} \ldots X^{\tilde{B}_{J}} g_{k}(\mathrm{u}),
\end{aligned}
$$

where the Pochhammer symbol is defined to be $(x)_{J}=\frac{\Gamma(x+J)}{\Gamma(x)}$. The explicit form of $K_{A}$ is given in (A.16), it satisfies $K_{A} K_{B}=K_{B} K_{A}$ (symmetric), $K^{A} K_{A}=0$ (traceless) and $X^{A} K_{A}=0$ (transverse), it allows us to implement the contraction between various STT tensors before we restrict to geodesics. Here we have also introduced the induced AdS metric $G_{A B}$ and the projection operator $G_{A}{ }^{B}$ in the embedding space:

$$
G_{A B}=\eta_{A B}+X_{A} X_{B}, \quad G_{A}^{B}=\delta_{A}^{B}+X_{A} X^{B}, \quad G_{A}{ }^{C} G_{C}^{B}=G_{A}^{B}, \quad G_{A}^{B} X_{B}=X^{A} G_{A}^{B}=0 .
$$

When contracting product of $G_{A}{ }^{B}$ with an arbitrary tensor in embedding space, such a tensor is then projected into the one satisfying the transverse condition, hence in the interior of hyperboloid corresponding to $\mathrm{AdS}_{d+1}$. Identical quantities can be defined for the other bulk vertex point with $X \rightarrow \tilde{X}$ and $i=1,2 \rightarrow i=3,4$. We can see that under the action of $K_{A}$ operators, (2.11) automatically satisfy the symmetric, traceless and transverse conditions.

It was shown in [14] that the bulk to bulk propagator can be related to the harmonic function $\Omega_{\Delta, J}(X, \tilde{X} ; W, \tilde{W})$ in $\operatorname{AdS}$ space as:

$$
\Omega_{\nu, J}(X, \tilde{X} ; W, \tilde{W})=\frac{i \nu}{2 \pi}\left(\Pi_{h+i \nu, J}(X, \tilde{X} ; W, \tilde{W})-\Pi_{h-i \nu, J}(X, \tilde{X} ; W, \tilde{W})\right)
$$




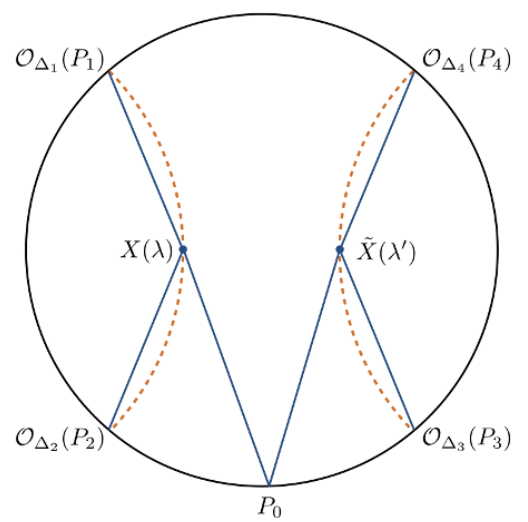

Figure 2. Cutting the four point scalar geodesic Witten diagram into the three point ones.

where $h=\frac{d}{2}$. We can invert this relation by considering following integral identity:

$$
\begin{aligned}
& \Pi_{\Delta, J}(X, \tilde{X} ; W, \tilde{W})=\int_{-\infty}^{+\infty} d \nu \frac{1}{\nu^{2}+(\Delta-h)^{2}} \Omega_{\nu, J}(X, \tilde{X} ; W, \tilde{W}) \\
& \quad=\int_{-\infty}^{+\infty} \frac{d \nu}{2 \pi i} \frac{\nu}{\nu^{2}+(\Delta-h)^{2}}\left(\Pi_{h-i \nu, J}(X, \tilde{X} ; W, \tilde{W})-\Pi_{h+i \nu, J}(X, \tilde{X} ; W, \tilde{W})\right),
\end{aligned}
$$

where in the last line as $\Pi_{h \mp i \nu, J}(X, \tilde{X} ; W, \tilde{W})$ only converges for $\operatorname{Im}(\nu) \rightarrow \pm \infty$, we have closed the integration contour in the upper (lower) half complex $\nu$-plane for first (second) term of the integrand. Moreover it was shown in [14] that it also admits following representations in terms of bulk to boundary propagators:

$$
\Omega_{\nu, J}(X, \tilde{X} ; W, \tilde{W})=\frac{\nu^{2}}{\pi J !(h-1)_{J}} \int_{\partial} d P_{0} \Pi_{h+i v, J}\left(X, P_{0} ; W, D_{Z_{0}}\right) \Pi_{h-i v, J}\left(\tilde{X}, P_{0} ; \tilde{W}, Z_{0}\right) .
$$

Here the spin- $J$ bulk to boundary propagator is:

$$
\Pi_{\Delta, J}(X, P ; W, Z)=\mathcal{C}_{\Delta, J} \frac{(2(X \cdot Z)(P \cdot W)-2(X \cdot P)(Z \cdot W))^{J}}{(-2 P \cdot X)^{\tau}}=\mathcal{C}_{\Delta, J} \frac{(2 X \cdot \mathrm{C} \cdot W)^{J}}{(-2 P \cdot X)^{\tau}},
$$

where $\tau=\Delta+J$ and for later purpose we have also defined the boundary anti-symmetric tensor

$$
\mathrm{C}^{A B}=Z^{A} P^{B}-P^{A} Z^{B}, \quad Z \cdot P=Z \cdot Z=0
$$

with $Z^{A}$ being the auxiliary polarization vector associated with boundary point $P^{A}$. Notice that $\mathrm{C}_{A B}$ hence bulk to boundary propagator (2.16) is manifestly invariant under the shift $Z^{A} \rightarrow Z^{A}+\alpha P^{A}$. The overall normalization constant is fixed to be:

$$
\mathcal{C}_{\Delta, J}=\frac{(J+\Delta-1) \Gamma(\Delta)}{2 \pi^{h}(\Delta-1) \Gamma(\Delta+1-h)} .
$$

In (2.15), we have also introduced the projection operator $D_{Z_{0}}:=D_{Z_{0}^{A}}$ defined in (A.17), which executes the tensor index contractions. Combining (2.13), (2.14) and (2.15), we 
can directly relate bulk to bulk and bulk to boundary propagators through the following "cutting identity": 4

$$
\begin{aligned}
\Pi_{\Delta, J}(X, \tilde{X} ; W, \tilde{W})= & \int_{-\infty}^{+\infty} d \nu \int_{\partial} d P_{0} \frac{\nu^{2}}{\pi J !(h-1)_{J}} \frac{1}{\nu^{2}+(\Delta-h)^{2}} \\
& \times \Pi_{h+i v, J}\left(X, P_{0} ; W, D_{Z_{0}}\right) \Pi_{h-i v, J}\left(\tilde{X}, P_{0} ; \tilde{W}, Z_{0}\right) .
\end{aligned}
$$

We will refer to the complex integration parameter $\nu$ as the "spectral parameter".

Given the relation (2.19), we can now use it to rewrite the bulk to bulk propagator entering (2.4). More explicitly as in (2.11), we can extract the STT tensor structures from (2.19) using the projection operator $K_{A}$ :

$$
\frac{1}{J !\left(\frac{d-1}{2}\right)_{J}} K_{A_{1} \ldots A_{J}} \Pi_{\Delta, J}(X, P ; W, Z)=\mathcal{C}_{\Delta, J} \frac{(2 X \cdot \mathrm{C})_{\left\{A_{1} \ldots A_{J}\right\}}}{(-2 P \cdot X)^{\tau}}
$$

where $(2 X \cdot \mathrm{C})_{A}=2 X^{B} \mathrm{C}_{B A}=-2 \mathrm{C}_{A B} X^{B}$ satisfies both transverse $(2 X \cdot \mathrm{C})_{A} X^{A}=0$ and traceless $\eta_{A B}(2 X \cdot \mathrm{C})^{A}(2 X \cdot \mathrm{C})^{B}=0$ properties. Moreover $(2 X \cdot \mathrm{C})_{B} G_{A}^{B}=(2 X \cdot \mathrm{C})_{A}$ simplifies the resultant expression.

Effectively upon the substitution, we have cut the four point geodesic Witten diagram into a pair of three point ones, and we call them "three point geodesic Witten diagrams" or "three point GWDs", see figure 3. We can now explicitly consider the general interaction vertex at $X(\lambda)$ (or $\tilde{X}\left(\lambda^{\prime}\right)$ ), which includes two massive scalar fields $\Phi_{1,2}(X)$ and a rank$J$ massive STT tensor field $\Xi_{J}(X)$, corresponding to the holographic duals of the CFT operators $\mathcal{O}_{\Delta_{1,2}}\left(P_{1,2}\right)$ and $\mathcal{O}_{h+i \nu, J}\left(P_{0}, Z_{0}\right)$ :

$$
g_{\Phi_{1} \Phi_{2} \Xi_{J}} \int_{X=X(\lambda)} d X \nabla^{C_{1}} \ldots \nabla^{C_{r}} \Phi_{1}(X) \nabla^{C_{r+1}} \ldots \nabla^{C_{J}} \Phi_{2}(X) \Xi(X)_{C_{1} \ldots C_{J}}
$$

where $r=1, \ldots, J$ encode all possible permutations of covariant derivatives and $g_{\Phi_{1} \Phi_{2} \Xi_{J}}$ is the coupling constant. In contrast with the usual three point Witten diagram, where the interaction point $X$ is integrated over the entire $\operatorname{AdS}_{d+1}$ space $X^{2}=-1$, here we restrict the interaction point only along the geodesic $\gamma_{12}: X=X(\lambda)$. Such that when we move the covariant derivatives using integration by parts and apply equation of motion, we need to carefully treat the boundary terms, this has interesting effect when we consider geodesic Witten diagrams involving external spinning fields.

If we now perform the integration along $\gamma_{12}$ first, the three point vertex $(2.21)$ generates the following integral: ${ }^{5}$

$$
\begin{gathered}
\int_{\gamma_{12}}(K \cdot \nabla)^{r} \frac{\mathcal{C}_{\Delta_{1}}}{\left(-2 P_{1} \cdot X\right)^{\Delta_{1}}}(K \cdot \nabla)^{J-r} \frac{\mathcal{C}_{\Delta_{2}}}{\left(-2 P_{2} \cdot X\right)^{\Delta_{2}}}\left[\mathcal{C}_{h+i \nu, J} \frac{\left(2 X \cdot C_{0} \cdot W\right)^{J}}{\left(-2 P_{0} \cdot X\right)^{h+i \nu+J}}\right] \\
\quad=2^{J}(-1)^{J-r} J !\left(\frac{d-1}{2}\right)_{J}\left(\Delta_{1}\right)_{r}\left(\Delta_{2}\right)_{J-r} \mathcal{C}_{\Delta_{1}} \mathcal{C}_{\Delta_{2}} \mathcal{C}_{h+i \nu, J} \beta_{\Delta_{12}, h+i \nu+J}\left[\begin{array}{ccc}
\Delta_{1} & \Delta_{2} & h+i \nu \\
0 & 0 & J \\
0 & 0 & 0
\end{array}\right] .
\end{gathered}
$$

\footnotetext{
${ }^{4}$ Here we refrain from using the terminology of closely related "split representation" to avoid confusion, as discussed in section 5, split representation of bulk to bulk propagator involves boundary to boundary propagators with lower spins.

${ }^{5}$ The detail of this calculation is relegated to (B.5).
} 


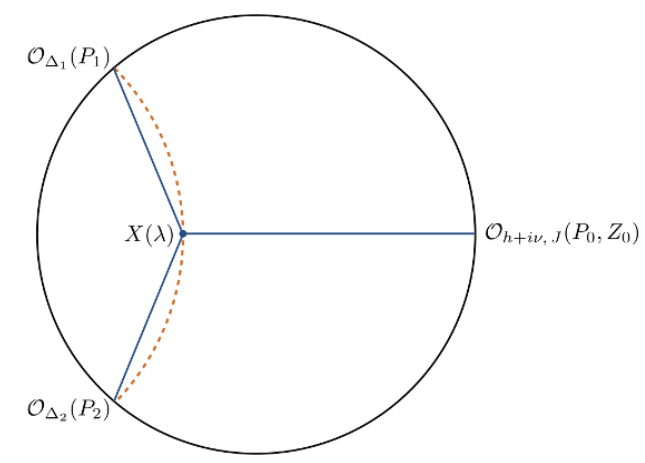

Figure 3. Three point scalar geodesic Witten diagram.

The lengthy calculation presented above requires some explanations. In the second line of (2.22), we have used the identity:

$$
(W \cdot \nabla)^{J-l} \frac{\left(2 X \cdot C_{0} \cdot W\right)^{l}}{\left(-2 P_{0} \cdot X\right)^{\Delta+l}}=(\Delta+l)_{J-l} \frac{\left(2 P_{0} \cdot W\right)^{J-l}\left(2 X \cdot C_{0} \cdot W\right)^{l}}{\left(-2 P_{0} \cdot X\right)^{\Delta+J}} .
$$

Restricting along the geodesic $\gamma_{12}$, we also have the following relations in the third line:

$$
\left(2 P_{1} \cdot G\right)_{A}=\left(-2 P_{1} \cdot X(\lambda)\right) \frac{d X_{A}(\lambda)}{d \lambda}, \quad\left(2 P_{2} \cdot G\right)_{A}=-\left(-2 P_{2} \cdot X(\lambda)\right) \frac{d X_{A}(\lambda)}{d \lambda},
$$

which yield the product of $\frac{d X(\lambda)}{d \lambda}$ and $\frac{d \tilde{X}\left(\lambda^{\prime}\right)}{d \lambda^{\prime}}$ appearing in (2.7). Finally in the last two lines, we introduced the independent tensor basis for three point functions defined in (3.3) and (3.5), and we have performed the integral using the result in appendix B. In particular, the $\nu$-dependent pre-factor is:

$$
\beta_{\Delta_{12}, h+i \nu+J}=\frac{\Gamma\left(\frac{h+i \nu+J+\Delta_{12}}{2}\right) \Gamma\left(\frac{h+i \nu+J-\Delta_{12}}{2}\right)}{2 \Gamma(h+i \nu+J)} .
$$

We can also consider analogous three point vertex to (2.21) along the geodesic $\gamma_{34}: \tilde{X}=$ $\tilde{X}(\lambda)$ for the holographic duals of $\mathcal{O}_{\Delta_{3,4}}\left(P_{3,4}\right)$ and $\mathcal{O}_{\frac{d}{2}-i \nu, J}\left(P_{0}, Z_{0}\right)$, and obtain the same tensor structure as in (2.22) with trivial substitution $\left(\Delta_{1}, \Delta_{2}, h+i \nu\right) \rightarrow\left(\Delta_{3}, \Delta_{4}, h-i \nu\right)$.

Gluing together the pair of resultant geodesic three point Witten diagrams for $\mathcal{O}_{\Delta_{1,2}}\left(P_{1,2}\right), \mathcal{O}_{\frac{d}{2}-i \nu, J}\left(P_{0}, Z_{0}\right)$ and $\mathcal{O}_{\Delta_{3,4}}\left(P_{3,4}\right), \mathcal{O}_{\frac{d}{2}-i \nu, J}\left(P_{0}, Z_{0}\right)$ given in $(2.22)$, by contracting their indices and integrating their common boundary point $P_{0}$, we obtained an integral representation of four point scalar geodesic Witten diagram $\mathcal{W}_{\Delta, l}\left(P_{i}\right)$ :

$$
\begin{aligned}
& \mathcal{S}_{\Delta_{1,2,3,4}}^{J, r, r^{\prime}} \int d P_{0} \int_{-\infty}^{\infty} d \nu \frac{\nu^{2} \mathcal{C}_{h+i \nu, J} \mathcal{C}_{h-i \nu, J} \beta_{\Delta_{12}, h+i \nu+J} \beta_{\Delta_{34}, h-i \nu+J}}{\nu^{2}+(\Delta-h)^{2}}\left[\begin{array}{ccc}
\Delta_{1} & \Delta_{2} & h+i \nu \\
0 & 0 & J \\
0 & 0 & 0
\end{array}\right] \cdot\left[\begin{array}{ccc}
\Delta_{3} & \Delta_{4} & h-i \nu \\
0 & 0 & J \\
0 & 0 & 0
\end{array}\right] \\
& =\frac{\mathcal{S}_{\Delta_{1,2,3,4}, r, r^{\prime}}}{2^{4} \pi^{d}} \int d P_{0} \int_{-\infty}^{\infty} d \nu \mathcal{B}_{d, J}(\nu) \frac{\mathcal{K}_{\Delta_{12}, \Delta_{34} ; J}(h+i \nu, h-i \nu)}{\nu^{2}+(\Delta-h)^{2}}\left[\begin{array}{ccc}
\Delta_{1} & \Delta_{2} & h+i \nu \\
0 & 0 & J \\
0 & 0 & 0
\end{array}\right] \cdot\left[\begin{array}{ccc}
\Delta_{3} & \Delta_{4} & h-i \nu \\
0 & 0 & J \\
0 & 0 & 0
\end{array}\right] .
\end{aligned}
$$


Here the overall constant is given by:

$$
\mathcal{S}_{\Delta_{1,2,3,4}^{J, r, r^{\prime}}}=\mathcal{C}_{\Delta_{1}} \mathcal{C}_{\Delta_{2}} \mathcal{C}_{\Delta_{3}} \mathcal{C}_{\Delta_{4}}\left[2^{J} J !\left(\frac{d-1}{2}\right)_{J}\right]^{2}\left(\Delta_{1}\right)_{r}\left(\Delta_{2}\right)_{J-r}\left(\Delta_{3}\right)_{r^{\prime}}\left(\Delta_{4}\right)_{J-r^{\prime}}
$$

The dot "." product between the two box tensor basis for the three point functions indicates that we have replaced $Z_{0}$ by $D_{Z_{0}}$ in the first term as in (2.19) to perform the index contractions. We have also defined the following short hand notations and composite functions:

$$
\begin{aligned}
\Gamma(x \pm b) & =\Gamma(x+b) \Gamma(x-b), & (x \pm b)_{J} & =(x+b)_{J}(x-b)_{J}, \\
\mathcal{B}_{h, J}(\nu) & =\frac{1}{(h-1 \pm i \nu)_{J} \Gamma( \pm i \nu)}, & \mathcal{K}_{\Delta_{12}, \Delta_{34} ; J}(x, y) & =\Gamma\left(\frac{x+J \pm \Delta_{12}}{2}\right) \Gamma\left(\frac{y+J \pm \Delta_{34}}{2}\right) .
\end{aligned}
$$

We can also deduce an analogous integral representation for the conformal partial wave $W_{\Delta, J}\left(P_{i}\right)$, which also involves the so-called "shadow operator" $\tilde{\mathcal{O}}_{\tilde{\Delta}, J}\left(P_{0}, Z_{0}\right)$ of the exchanged operator $\mathcal{O}_{\Delta, J}\left(P_{0}, Z_{0}\right)[8,9]$, carrying the scaling dimension $\tilde{\Delta}=d-\Delta$ and the same spin $J$. Our starting point is the equation (3.25) of [8], which relates the linear combination of the conformal block $G_{\mathcal{O}_{\Delta, J}}(u, v)$ and its shadow $G_{\tilde{\mathcal{O}}_{\tilde{\Delta}, J}}(u, v)$, with an integral containing a pair of three point functions involving $\mathcal{O}_{\Delta, J}$ and $\tilde{\mathcal{O}}_{\tilde{\Delta}, J}$. By multiplying the appropriate pre-factors as in (2.1), we can deduce the following equation for the conformal partial wave and its shadow: ${ }^{6}$

$$
\begin{aligned}
& (-1)^{J} \frac{\mathcal{K}_{\Delta_{12}, \Delta_{34}, J}(h+i \nu, h-i \nu)}{\pi^{\frac{d}{2}}} \int d P_{0}\left[\begin{array}{ccc}
\Delta_{1} & \Delta_{2} & h+i \nu \\
0 & 0 & J \\
0 & 0 & 0
\end{array}\right] \cdot\left[\begin{array}{ccc}
\Delta_{3} & \Delta_{4} & h-i \nu \\
0 & 0 & J \\
0 & 0 & 0
\end{array}\right] \\
& =\frac{1}{2^{J} c_{J}}\left(\frac{\mathcal{K}_{\Delta_{12}, \Delta_{34}, J}(h+i \nu, h+i \nu)}{k_{h-i \nu, J}} W_{\mathcal{O}_{h+i \nu, J}}\left(P_{i}\right)+\frac{\mathcal{K}_{\Delta_{12}, \Delta_{34}, J}(h-i \nu, h-i \nu)}{k_{h+i \nu, J}} W_{\tilde{\mathcal{O}}_{h-i \nu, J}}\left(P_{i}\right)\right),
\end{aligned}
$$

where we have set $\Delta=h+i \nu$ and defined:

$$
c_{J}=\frac{\left(\frac{d-2}{2}\right)_{J}}{(d-2)_{J}} \quad k_{\Delta, J}=\frac{\Gamma(\Delta-1) \Gamma(d-\Delta+J)}{\Gamma(\Delta-1+J) \Gamma(\Delta-h)} .
$$

Now to revert the relation $(2.30)$ and extract $W_{\mathcal{O}_{\Delta, J}}\left(P_{i}\right)$, we first multiply both sides with $\frac{\mathcal{B}_{h, J}(\nu)}{\nu^{2}+(h-\Delta)^{2}}$ and integrate over $\nu$, clearly the l.h.s. is now proportional to $(2.26)$, while from r.h.s. we obtained the following integral:

$\frac{1}{2^{J} c_{J}} \int_{-\infty}^{\infty} d \nu \frac{1}{\nu^{2}+(h-\Delta)^{2}}\left(f_{\Delta_{12}, \Delta_{34}, J}(h+i \nu) W_{\mathcal{O}_{h+i \nu, J}}\left(P_{i}\right)+f_{\Delta_{12}, \Delta_{34}, J}(h-i \nu) W_{\tilde{\mathcal{O}}_{h-i \nu, J}}\left(P_{i}\right)\right)$,

\footnotetext{
${ }^{6}$ We can verify by direct computation that the embedding space building blocks $\mathrm{V}_{0,12}$ and $\mathrm{V}_{0,34}$ can be projected into physical space as $\mathrm{V}_{0,12}=\frac{x_{01}^{2} x_{02}^{2}}{x_{12}^{2}} X_{\nu} z_{0}^{\nu}$ and $\mathrm{V}_{0,34}=-\frac{x_{03}^{2} x_{04}^{2}}{x_{34}^{2}} \bar{X}_{\nu} z_{0}^{\nu}$, where $X^{\mu}$ and $\bar{X}^{\mu}$ are the vectors defined in equations (3.6) of [8]. We can then make identifications between the three point functions in physical space and the embedding space box tensor basis as in (2.30).
} 
where

$$
f_{\Delta_{12}, \Delta_{34}, J}(h \pm i \nu)=\frac{\mathcal{K}_{\Delta_{12}, \Delta_{34}, J}(h \pm i \nu, h \pm i \nu)}{\Gamma( \pm i \nu) \Gamma(h \pm i \nu+J)(h \pm i \nu-1)_{J}} .
$$

In the first integrand of (2.32), since $W_{\mathcal{O}_{h+i \nu, J}}\left(P_{i}\right) \rightarrow 0$ as $\operatorname{Im}(\nu) \rightarrow-\infty$, assuming $\Delta>h$, we close the contour in the lower half plane to pick up the residue at $\nu=-i(\Delta-h)$, similarly for the second integrand of (2.32), we can close the contour in the upper half plane to pick up the residue at $\nu=+i(\Delta-h)$. Moreover one can check that provided

$$
J+h>\left|\Delta_{12}\right|,\left|\Delta_{34}\right|
$$

is satisfied, the factor $f_{\Delta_{12}, \Delta_{34}, J}(h \pm i \nu)$ does not contain any additional poles in the lower/upper half plane. ${ }^{7}$ Collecting all the factors we see that the conformal partial wave then takes the following integral representation: ${ }^{8}$

$$
\begin{aligned}
W_{\Delta, J}\left(P_{i}\right)= & F_{\Delta_{12}, \Delta_{34}, J}(\Delta) \int d P_{0} \int_{-\infty}^{\infty} d \nu \mathcal{B}_{h, J}(\nu) \frac{\mathcal{K}_{\Delta_{12}, \Delta_{34}, J}(h+i \nu, h-i \nu)}{\nu^{2}+(\Delta-h)^{2}} \\
& \times\left[\begin{array}{ccc}
\Delta_{1} & \Delta_{2} & h+i \nu \\
0 & 0 & J \\
0 & 0 & 0
\end{array}\right] \cdot\left[\begin{array}{ccc}
\Delta_{3} & \Delta_{4} & h-i \nu \\
0 & 0 & J \\
0 & 0 & 0
\end{array}\right]
\end{aligned}
$$

where $F_{\Delta_{12}, \Delta_{34}, J}(\Delta)=\frac{(-2)^{J}(\Delta-h) c_{J}}{2 \pi^{h+1} f_{\Delta_{12}, \Delta_{34}, J}(\Delta)}$. We see that up to overall constants the integral representation of four point scalar geodesic Witten diagram (2.26) precisely matches with the integral representation of the scalar conformal partial wave (2.35). This provides an alternative proof of the results in [1]. In particular, we have done so by using the relation (2.19) to build the scalar four point geodesic Witten diagram using three point ones, through the integration with the corresponding measure as in (2.26). This is completely analogous to how we construct the conformal blocks using three point functions.

Closely related computation has been done in [14] and [15], which builds the four point Witten diagrams from the three point ones, ${ }^{9}$ it is somewhat expected that the three point Witten and geodesic Witten diagrams for scalar-scalar-spin- $J$ exchange are both proportional to the same tensor structure, as there is only single one available. The crucial difference here however is the different $\nu$-dependent pre-factors generated through integration over entire $\mathrm{AdS}_{d+1}$ and only along geodesics. The pre-factor for three point Witten diagrams, upon integrating with the same $\frac{1}{\nu^{2}+(\Delta-h)^{2}}$ typically yields conformal blocks for operator $\mathcal{O}_{\Delta, J}$ plus infinite towers of double trace operator $\mathcal{O}_{\Delta_{m}^{(12)}, l}$ and $\mathcal{O}_{\Delta_{n}^{(34)}, l}$ where $0 \leq l<J$ and the dimensions $\Delta_{m, l}^{(12)}$ and $\Delta_{m, l}^{(34)}$ are defined in (5.21). While the corresponding pre-factor for three point geodesic Witten diagram (2.25) does not contain these infinite double trace operator poles, such that upon integration (2.26) we precisely only have conformal block for $\mathcal{O}_{\Delta, J}$ exchange. In section 5 , we will start from the so-called "split representation" of the four point Witten diagram to recover their decompositions into four geodesic Witten diagrams for single and double trace operator exchanges with arbitrary spins. We will see that the three point geodesic Witten diagrams play the role of building block for various four point geodesic Witten diagrams.

\footnotetext{
${ }^{7}$ This is obviously satisfied by identical scalars $\Delta_{12}=\Delta_{34}=0$.

${ }^{8}$ Special case of this expression for identical scalars was also obtained in [27].

${ }^{9}$ More comprehensive analysis can also be found in [27].
} 


\section{Spinning three point functions and conformal blocks}

Having demonstrated how the integral representation of scalar conformal partial waves can be directly realized through cutting up the four point scalar GWDs, and identify the resultant three point GWDs with the three point correlation functions, this procedure becomes even more useful when systematically constructing the holographic dual configuration for conformal partial waves/conformal blocks for external operators carrying arbitrary integer spins. Here we will restrict ourselves here to only the exchange of symmetric traceless field, as in the case of scalar conformal partial waves we just reviewed. Even though there can be additional exchange channels involving mixed tensor fields (see e.g. [24-26]), we leave the detailed holographic analysis to the future work.

Let us begin by reviewing CFT side of the story, this was done throughly in $[10,11]$. The external primary operators with scaling dimension $\Delta_{i}$ and spin $l_{i}$ are labeled as $\mathcal{O}_{\Delta_{i}, l_{i}}\left(P_{i}, Z_{i}\right)$, where $P_{i}$ is the position in the embedding space as before and $Z_{i}$ is the auxiliary polarization vector. Such that $\mathcal{O}_{\Delta_{i}, l_{i}}\left(P_{i}, Z_{i}\right)$ is a homogenous polynomial in $Z_{i}$ of degree $l_{i}$, and we can recover the STT tensor field in embedding space through differential operator $D_{Z_{i}}$ defined in (A.17). We again collect the relevant details about the embedding space representatives for $d$-dimensional tensors in the appendix A.

The three point correlation functions involving $\left\{\mathcal{O}_{\Delta_{i}, l_{i}}\left(P_{i}, Z_{i}\right)\right\}$ are crucial building blocks for higher point correlation functions, their form can be completely fixed by conformal symmetries manifest in the embedding space, which lead to the classifications in $[10,11]$ :

$$
\begin{aligned}
< & \mathcal{O}_{\Delta_{1}, l_{1}}\left(P_{1}, Z_{1}\right) \mathcal{O}_{\Delta_{2}, l_{2}}\left(P_{2}, Z_{2}\right) \mathcal{O}_{\Delta_{3}, l_{3}}\left(P_{3}, Z_{3}\right)> \\
& =\sum_{n_{12}, n_{13}, n_{23} \geq 0} \lambda_{n_{12}, n_{13}, n_{23}}\left[\begin{array}{ccc}
\Delta_{1} & \Delta_{2} & \Delta_{3} \\
l_{1} & l_{2} & l_{3} \\
n_{23} & n_{13} & n_{12}
\end{array}\right]+\mathcal{O}\left(Z_{i}^{2}, Z_{i} \cdot P_{i}\right) .
\end{aligned}
$$

Here $\lambda_{n_{12}, n_{13}, n_{23}}$ are theory dependent constant expansion coefficients, and in addition to the integer spins $\left\{l_{i}\right\}$, we have also introduced triplet of non-negative integers $\left\{n_{12}, n_{13}, n_{23}\right\}$ satisfying the following constraint:

$$
m_{1}=l_{1}-n_{12}-n_{13} \geq 0, \quad m_{2}=l_{2}-n_{12}-n_{23} \geq 0 \quad m_{3}=l_{3}-n_{13}-n_{23} \geq 0 .
$$

The elementary structures of the three point correlation function, which we shall call "box tensor basis" are then given by:

$$
\left[\begin{array}{ccc}
\Delta_{1} & \Delta_{2} & \Delta_{3} \\
l_{1} & l_{2} & l_{3} \\
n_{23} & n_{13} & n_{12}
\end{array}\right]=\frac{\mathrm{V}_{1,23}^{m_{1}} \mathrm{~V}_{2,31}^{m_{2}} \mathrm{~V}_{3,12}^{m_{3}} \mathrm{H}_{12}^{n_{12}} \mathrm{H}_{13}^{n_{13}} \mathrm{H}_{23}^{n_{23}}}{\left(P_{12}\right)^{\frac{1}{2}\left(\tau_{1}+\tau_{2}-\tau_{3}\right)}\left(P_{13}\right)^{\frac{1}{2}\left(\tau_{1}+\tau_{3}-\tau_{2}\right)}\left(P_{23}\right)^{\frac{1}{2}\left(\tau_{2}+\tau_{3}-\tau_{1}\right)}} .
$$

Here we have defined the six linearly independent tensor basis for three operators with integer spins:

$$
\begin{aligned}
\mathrm{H}_{i j} & =-2\left[\left(Z_{i} \cdot Z_{j}\right)\left(P_{i} \cdot P_{j}\right)-\left(Z_{i} \cdot P_{j}\right)\left(Z_{j} \cdot P_{i}\right)\right]=-\operatorname{Tr}\left(\mathrm{C}_{i} \cdot \mathrm{C}_{j}\right), \\
\mathrm{V}_{i, j k} & =\frac{\left(P_{j} \cdot Z_{i}\right)\left(P_{i} \cdot P_{k}\right)-\left(P_{j} \cdot P_{i}\right)\left(Z_{i} \cdot P_{k}\right)}{\left(P_{j} \cdot P_{k}\right)}=\frac{\left(P_{j} \cdot \mathrm{C}_{i} \cdot P_{k}\right)}{\left(P_{j} \cdot P_{k}\right)}, \quad i, j, k=1,2,3
\end{aligned}
$$


Notice that $\mathrm{H}_{j k}$ is symmetric and $\mathrm{V}_{i, j k}$ is anti-symmetric under the exchange of $j$ and $k$ indices, such that we only have altogether six independent basis. They combine to form transverse polynomial of degree $l_{i}$ in each $Z_{i}$ (also each $P_{i}$ ) in the numerator of (3.3), the tensorial structure of (3.3) is revealed through the action of $D_{Z_{i}}$ operators. The number of the set of non-negative integers satisfying (3.2) is the possible elementary structures listed in (3.3), for $l_{3} \geq l_{2} \geq l_{1}$ and $p=\max \left(0, l_{1}+l_{2}-l_{3}\right)$, it is given by:

$$
N\left(l_{1}, l_{2}, l_{3}\right)=\frac{\left(l_{1}+1\right)\left(l_{1}+2\right)\left(3 l_{2}-l_{1}+3\right)}{6}-\frac{p(p+2)(2 p+5)}{24}-\frac{1-(-1)^{p}}{16} .
$$

The remaining terms labeled $\mathcal{O}\left(Z_{i}^{2}, Z_{i} \cdot P_{i}\right)$ arise from $\left(\mathrm{C}_{i} \cdot \mathrm{C}_{i}\right),\left(\mathrm{C}_{i} \cdot P_{i}\right)$ or $\left(\mathrm{C}_{i} \cdot Z_{i}\right)$ types of contractions which are not independent and can be determined when taking into account of light cone and transversality conditions.

Another very useful basis for expressing the structures of three point functions involve the following differential operators:

$$
\begin{aligned}
& \mathrm{D}_{11}=\left(\left(P_{1} \cdot P_{2}\right) Z_{1}^{A}-\left(Z_{1} \cdot P_{2}\right) P_{1}^{A}\right) \frac{\partial}{\partial P_{2}^{A}}+\left(\left(P_{1} \cdot Z_{2}\right) Z_{1}^{A}-\left(Z_{1} \cdot Z_{2}\right) P_{1}^{A}\right) \frac{\partial}{\partial Z_{2}^{A}} \\
& \mathrm{D}_{12}=\left(\left(P_{1} \cdot P_{2}\right) Z_{1}^{A}-\left(Z_{1} \cdot P_{2}\right) P_{1}^{A}\right) \frac{\partial}{\partial P_{1}^{A}}+\left(\left(P_{2} \cdot Z_{1}\right) Z_{1}^{A}\right) \frac{\partial}{\partial Z_{1}^{A}}, \\
& \mathrm{D}_{22}=\left(\left(P_{1} \cdot P_{2}\right) Z_{2}^{A}-\left(Z_{2} \cdot P_{1}\right) P_{2}^{A}\right) \frac{\partial}{\partial P_{1}^{A}}+\left(\left(P_{2} \cdot Z_{1}\right) Z_{2}^{A}-\left(Z_{1} \cdot Z_{2}\right) P_{2}^{A}\right) \frac{\partial}{\partial Z_{1}^{A}}, \\
& \mathrm{D}_{21}=\left(\left(P_{1} \cdot P_{2}\right) Z_{2}^{A}-\left(Z_{2} \cdot P_{1}\right) P_{2}^{A}\right) \frac{\partial}{\partial P_{2}^{A}}+\left(\left(P_{1} \cdot Z_{2}\right) Z_{2}^{A}\right) \frac{\partial}{\partial Z_{2}^{A}},
\end{aligned}
$$

and they only have the following non-vanishing commutators:

$$
\begin{aligned}
& {\left[\mathrm{D}_{11}, \mathrm{D}_{22}\right]=\frac{\mathrm{H}_{12}}{2}\left(Z_{1} \cdot \frac{\partial}{\partial Z_{1}}-Z_{2} \cdot \frac{\partial}{\partial Z_{2}}+P_{1} \cdot \frac{\partial}{\partial P_{1}}-P_{2} \cdot \frac{\partial}{\partial P_{2}}\right),} \\
& {\left[\mathrm{D}_{12}, \mathrm{D}_{21}\right]=\frac{\mathrm{H}_{12}}{2}\left(Z_{1} \cdot \frac{\partial}{\partial Z_{1}}-Z_{2} \cdot \frac{\partial}{\partial Z_{2}}-P_{1} \cdot \frac{\partial}{\partial P_{1}}+P_{2} \cdot \frac{\partial}{\partial P_{2}}\right),}
\end{aligned}
$$

while all other commutators vanish, including $\left[\mathrm{D}_{i j}, \mathrm{H}_{12}\right]=0$. We shall express such differential basis using curly brackets, and they are defined through the following relations:

$$
\begin{aligned}
\left\{\begin{array}{ccc}
\Delta_{1} & \Delta_{2} & \Delta_{3} \\
l_{1} & l_{2} & l_{3} \\
n_{23} & n_{13} & n_{12}
\end{array}\right\} & =\mathrm{H}_{12}^{n_{12}} \mathrm{D}_{12}^{n_{13}} \mathrm{D}_{21}^{n_{23}} \mathrm{D}_{11}^{m_{1}} \mathrm{D}_{22}^{m_{2}} \Sigma^{l_{1}+n_{23}-n_{13}, l_{2}+n_{13}-n_{23}}\left[\begin{array}{ccc}
\Delta_{1} & \Delta_{2} & \Delta_{3} \\
0 & 0 & l_{3} \\
0 & 0 & 0
\end{array}\right], \\
& =\mathrm{H}_{12}^{n_{12}} \mathrm{D}_{12}^{n_{13}} \mathrm{D}_{21}^{n_{23}} \mathrm{D}_{11}^{m_{1}} \mathrm{D}_{22}^{m_{2}}\left[\begin{array}{ccc}
\tilde{\tau}_{1} & \tilde{\tau}_{2} & \Delta_{3} \\
0 & 0 & l_{3} \\
0 & 0 & 0
\end{array}\right],
\end{aligned}
$$

where the shift operators $\Sigma^{a, b}$ which shifts the scaling dimensions $\left(\Delta_{1}, \Delta_{2}\right)$ to $\left(\Delta_{1}+a, \Delta_{2}+\right.$ $b)$, such that $\tilde{\tau}_{1}=\tau_{1}+\left(n_{23}-n_{13}\right)$ and $\tilde{\tau}_{2}=\tau_{2}+\left(n_{13}-n_{23}\right)$. Notice that for given integer spins $\left\{l_{1}, l_{2}, l_{3}\right\},(3.13)$ are also labeled by triplet of non-negative integers $\left\{n_{12}, n_{13}, n_{23}\right\}$ satisfying (3.2), we therefore have equal number $N\left(l_{1}, l_{2}, l_{3}\right)$ of differential basis (3.13) as 
in the original box basis (3.3), and they are related by linear transformation with constant coefficients.

In contrast with the box basis (3.3), where we can cyclicly permute the three primary operators involved, in the differential basis we break this cyclicity such that the differential operators (3.7)-(3.10) only act on $\left(P_{1,2}, Z_{1,2}\right)$, moreover the remaining box tensor structure in the r.h.s. of (3.13) is precisely the one arising in the integral representation of conformal partial wave (2.35) after identifying $\left(\Delta_{3}, l_{3}\right)$ with $(h+i \nu, J)$. We can therefore regard the remaining primary operator as the internal exchange operator when constructing the four point correlation function, this allows us to relate four point correlation functions for operators with spins:

$$
<\mathcal{O}_{\Delta_{1}, l_{1}}\left(P_{1}, Z_{1}\right) \mathcal{O}_{\Delta_{2}, l_{2}}\left(P_{2}, Z_{2}\right) \mathcal{O}_{\Delta_{3}, l_{3}}\left(P_{3}, Z_{3}\right) \mathcal{O}_{\Delta_{4}, l_{4}}\left(P_{4}, Z_{4}\right)>
$$

with the scalar ones. More explicitly, unlike scalar case (2.1) whose conformal partial wave for a given exchanged operator $\mathcal{O}_{\Delta, J}$ can be packaged into a single scalar function of cross-ratios; the conformal partial wave for (3.14) for a given exchange operator consists of multiple terms each with independent tensor structures. When restricting to only the exchange of symmetric traceless operators, we can construct it by fusing the differential basis for a pair of three point correlation functions involving primary operators $\mathcal{O}_{\Delta_{1,2}, l_{1,2}}, \mathcal{O}_{\Delta, J}$ and $\mathcal{O}_{\Delta_{3,4}, l_{3,4}}, \tilde{\mathcal{O}}_{d-\Delta, J}$ and the resultant conformal partial wave schematically contains the following tensor structures:

$$
W_{\mathcal{O}_{\Delta, J}}^{\left\{n_{10}, n_{20}, n_{12}\right\} ;\left\{n_{30}, n_{40}, n_{34}\right\}}\left(P_{i}, Z_{i}\right):=\mathcal{D}_{\text {Left }}^{n_{10}, n_{20}, n_{12}} \mathcal{D}_{\text {Right }}^{n_{30}, n_{40}, n_{34}} W_{\mathcal{O}_{\Delta, J}}\left(P_{i}\right) .
$$

Here the composite operators are given by:

$$
\begin{aligned}
& \mathcal{D}_{\text {Left }}^{n_{10}, n_{20}, n_{12}}=\mathrm{H}_{12}^{n_{12}} \mathrm{D}_{12}^{n_{10}} \mathrm{D}_{21}^{n_{20}} \mathrm{D}_{11}^{m_{1}} \mathrm{D}_{22}^{m_{2}} \Sigma^{l_{1}+n_{20}-n_{10}, l_{2}-n_{20}+n_{10}}, \\
& \mathcal{D}_{\text {Right }}^{n_{30}, n_{40}, n_{34}}=\mathrm{H}_{34}^{n_{34}} \mathrm{D}_{34}^{n_{30}} \mathrm{D}_{43}^{n_{40}} \mathrm{D}_{33}^{m_{3}} \mathrm{D}_{44}^{m_{4}} \Sigma^{l_{3}+n_{40}-n_{30}, l_{4}-n_{40}+n_{30}}
\end{aligned}
$$

They are now labeled by two sets of triplet of integers $\left\{n_{10}, n_{20}, n_{12}\right\}$ and $\left\{n_{30}, n_{40}, n_{34}\right\}$ satisfying (3.2), and we have denoted the exchanged operator as $\mathcal{O}_{\Delta_{0}, l_{0}} \equiv \mathcal{O}_{\Delta, J}$. Summing over all $N\left(l_{1}, l_{2}, l_{0}\right) \times N\left(l_{3}, l_{4}, l_{0}\right)$ possible tensor structures listed in (3.15), we can express the resultant conformal partial wave for $\mathcal{O}_{\Delta, J}$ as:

$$
\begin{gathered}
\sum_{\left\{n_{10}, n_{20}, n_{12}\right\}\left\{n_{30}, n_{40}, n_{34}\right\}} W_{\mathcal{O}_{\Delta, J}}^{\left\{n_{10}, n_{20}, n_{12}\right\} ;\left\{n_{30}, n_{40}, n_{34}\right\}}\left(P_{i}, Z_{i}\right) \\
=\left(\frac{P_{24}}{P_{14}}\right)^{\frac{\tau_{1}-\tau_{2}}{2}}\left(\frac{P_{14}}{P_{13}}\right)^{\frac{\tau_{3}-\tau_{4}}{2}} \frac{\sum_{k} f_{k}(u, v) Q^{(k)}\left(P_{i} ; Z_{i}\right)}{\left(P_{12}\right)^{\frac{\tau_{1}+\tau_{2}}{2}}\left(P_{34}\right)^{\frac{\tau_{3}+\tau_{4}}{2}}} .
\end{gathered}
$$

Here $Q^{(k)}\left(P_{i}, Z_{i}\right)$ are transverse polynomials of degree $l_{i}$ in $Z_{i}$ and can be built from $\mathrm{H}_{12}$, $\mathrm{H}_{34}$ and $\mathrm{V}_{i, j k}$ given in (3.4) and (3.5), now with $i, j, k=1,2,3,4 . \quad f_{k}(u, v)$ denote the functions of purely cross ratio $(u, v)$ which can be obtained by mechanical differentiations involving $\mathrm{D}_{i j}$ operators, and they consist of derivatives of the scalar conformal block for the same exchange operator $G_{\mathcal{O}_{\Delta, J}}(u, v)$ with respect to cross ratios $(u, v)$. It is interesting to note that all the differential operators $\mathrm{D}_{i j}$ (3.13) only act on the external position 
and polarization vectors $\left(P_{i}, Z_{i}\right)$, we can thus readily obtain conformal partial waves for spinning primary operators in terms of the scalar ones.

We can also easily deduce the integral representation for the spinning conformal blocks from the one for scalar conformal block [9]. This amounts to simply replacing the box tensor structures for $\mathcal{O}_{\Delta_{1,2}}\left(P_{1,2}\right)$ and $\mathcal{O}_{h+i \nu, J}\left(P_{0}, Z_{0}\right)$ three point function appearing in (2.35) with the differential basis (3.13), and identify $\left(\Delta, l_{3}\right)$ with $(h+i \nu, J)$, and similarly for the $\mathcal{O}_{\Delta_{3,4}}\left(P_{3,4}\right)$ and the shadow $\tilde{\mathcal{O}}_{h-i \nu, J}\left(P_{0}, Z_{0}\right)$ three point function. The result is thus:

$$
\begin{aligned}
& W_{\mathcal{O}_{\Delta, J}\left\{n_{10}, n_{20}, n_{12}\right\} ;\left\{n_{30}, n_{40}, n_{34}\right\}}\left(P_{i}, Z_{i}\right) \\
& \propto \hat{\mathcal{D}}_{\text {Left }}^{n_{10}, n_{20}, n_{12}} \hat{\mathcal{D}}_{\mathrm{Right}}^{n_{30}, n_{40}, n_{34}} \int d P_{0} \int_{-\infty}^{\infty} d \nu \mathcal{B}_{h, J}(\nu) \frac{\mathcal{K}_{\tilde{\tau}_{12}, \tilde{\tau}_{34}, J}(h+i \nu, h-i \nu)}{\nu^{2}+(\Delta-h)^{2}} \\
& \quad \times\left[\begin{array}{ccc}
\tilde{\tau}_{1} & \tilde{\tau}_{2} & h+i \nu \\
0 & 0 & J \\
0 & 0 & 0
\end{array}\right] \cdot\left[\begin{array}{ccc}
\tilde{\tau}_{3} & \tilde{\tau}_{4} & h-i \nu \\
0 & 0 & J \\
0 & 0 & 0
\end{array}\right], \\
& =\int d P_{0} \int_{-\infty}^{\infty} d \nu \mathcal{B}_{h, J}(\nu) \frac{\mathcal{K}_{\tilde{\tau}_{12}, \tilde{\tau}_{34}, J}(h+i \nu, h-i \nu)}{\nu^{2}+(\Delta-h)^{2}}\left\{\begin{array}{ccc}
\Delta_{1} & \Delta_{2} & h+i \nu \\
l_{1} & l_{2} & J \\
n_{20} & n_{10} & n_{12}
\end{array}\right\} \cdot\left\{\begin{array}{ccc}
\Delta_{3} & \Delta_{4} & h-i \nu \\
l_{3} & l_{4} & J \\
n_{40} & n_{30} & n_{34}
\end{array}\right\} .
\end{aligned}
$$

where $\hat{\mathcal{D}}_{\text {Left }}$ and $\hat{\mathcal{D}}_{\text {Right }}$ are defined similarly to (3.16) and (3.17) up the shift operators $\Sigma^{a, b}$, whose action on the scaling dimensions has been absorbed into the integral. This makes clear that we have a integral representation of a scalar conformal partial wave in the second line above with $\left\{\Delta_{i}\right\} \rightarrow\left\{\tilde{\tau}_{i}\right\}$, followed by the action of differential operators $\hat{\mathcal{D}}_{\text {Left }}$ and $\hat{\mathcal{D}}_{\text {Right }}$. We will see in the next section that exactly the same integral representation naturally appearing in the holographic reconstruction of the spinning conformal blocks.

\section{Spinning conformal partial waves from Anti-de Sitter space}

Let us begin holographic reconstruction of spinning conformal partial waves given in terms of the basis in (3.15). Our strategy is simple, given the success of cutting up the four point geodesic Witten diagram into three point ones to reproduce the integral representation of scalar conformal blocks reviewed in section 2, we will again first consider the geodesic three point Witten diagrams involving the holographic duals of spinning primary operators $\mathcal{O}_{\Delta_{1,2}, l_{1,2}}\left(P_{1,2}, Z_{1,2}\right)$ and the operator $\mathcal{O}_{\Delta_{0}, l_{0}}\left(P_{0}, Z_{0}\right)$ in their operator product expansion. We will first prove that all possible conformally invariant three point interaction vertices, when restricting along geodesic, can be expressed as linear combinations of the box tensor basis given in (3.3), where the expansion coefficients only depend on scalar products $\left(P_{i} \cdot P_{j}\right)$, $i, j=1,2,3,4$. Given the box tensor basis can be cast into differential tensor basis (3.13) by linear transformations, moreover the composite differential operators (3.16) and (3.17) commute with the integration over boundary point $P_{0}$, we can apply the same gluing procedure as in the scalar case to obtain the holographic reconstruction of the various integral representation of spinning conformal partial waves schematically given in (3.15).

Working again in the $d+2$ dimensional embedding space, let us begin by considering all possible non-vanishing Lorentz invariant contractions among three bulk to boundary 
propagators of scale dimensions $\Delta_{1,2,0}$ and spins $l_{1,2,0}$ with metric tensor $\eta_{A B}$ and arbitrary number of covariant derivatives $\nabla_{A}$. They can appear in the integrand for three point functions generated by all possible three point interaction vertices, whose explicit form we will discuss momentarily. Using the equation $(2.23)$ and $\nabla_{A}\left(X \cdot \mathrm{C}_{i}\right)_{B}=G_{A}^{A^{\prime}} G_{B}^{B^{\prime}} \mathrm{C}_{i A^{\prime} B^{\prime}}$, we can see that the numerator in a generic term consists of all possible invariant contractions among $P_{i}^{A}, \mathrm{C}_{i}^{A B}$ and $X^{A}$;

$$
\int_{X=X(\lambda)} d \lambda \frac{\mathbb{Q}\left(\left\{P_{i}, Z_{i}, X\right\}\right)}{\left(P_{1} \cdot X\right)^{\tau_{1}}\left(P_{2} \cdot X\right)^{\tau_{2}}\left(P_{0} \cdot X\right)^{\tau_{0}}} .
$$

Restricting the bulk coordinate $X$ along the geodesic $\gamma_{12}$ given in (2.5), the polynomial $\mathbb{Q}$ now only depends only $P_{1,2}$ and $Z_{1,2}$. Moreover $\mathbb{Q}$ is invariant under the shift $Z_{i} \rightarrow Z_{i}+\alpha P_{i}$, as $\mathbb{Q}$ depends on $Z_{i}$ only through $\mathrm{C}_{i}$ and $\mathrm{C}_{i}$ is invariant under such a shift. According to the discussion in [11] or done in more details in appendix $\mathrm{D}$, it can be represented by using only $\mathrm{H}_{i j}$ and $\mathrm{V}_{i j k}$ defined in (3.4) and (3.5) respectively. Therefore three point geodesic Witten diagram with an arbitrary interaction gives a linear combination of the box tensor basis (3.3).

Next we would like to consider the complete three point interaction vertices involving three symmetric traceless fields in $\mathrm{AdS}_{d+1}$ for the three point geodesic Witten diagrams, in terms of the embedding coordinates, it can be succinctly written in the following form:

$$
\mathcal{V}_{l_{1}, l_{2}, l_{0}}=\sum_{0 \leq \mathrm{n}_{r} \leq l_{r}} g_{l_{1}, l_{2}, l_{0}}^{\mathrm{n}_{1}, \mathrm{n}_{2}, \mathrm{n}_{0}} \mathcal{J}_{l_{1}, l_{2}, l_{0}}^{\mathrm{n}_{1}, \mathrm{n}_{2}, \mathrm{n}_{0}}\left(\mathcal{T}^{r}\right), \quad r=1,2,3 .
$$

Here $\left\{g_{l_{1}, l_{2}, l_{0}}^{\mathrm{n}_{1}, \mathrm{n}_{2}, \mathrm{n}_{0}}\right\}$ are the theory dependent bulk coupling constants which can be eventually related the CFT OPE coefficients, and the integers $\left\{\mathrm{n}_{1}, \mathrm{n}_{2}, \mathrm{n}_{0}\right\}$ need to satisfy the conditions: ${ }^{10}$

$$
l_{1}-\mathrm{n}_{2}-\mathrm{n}_{0} \geq 0, \quad l_{2}-\mathrm{n}_{1}-\mathrm{n}_{0} \geq 0, \quad l_{0}-\mathrm{n}_{1}-\mathrm{n}_{2} \geq 0 .
$$

While the interaction vertices along the geodesic $\gamma_{12}$ are parameterized by:

$$
\begin{aligned}
\mathcal{J}_{l_{1}, l_{2}, l_{0}}^{\mathrm{n}_{1}, \mathrm{n}_{2}, \mathrm{n}_{0}}\left(\mathcal{T}^{r}\right)= & \mathcal{Y}_{1}^{l_{1}-\mathrm{n}_{2}-\mathrm{n}_{0}} \mathcal{Y}_{2}^{l_{2}-\mathrm{n}_{0}-\mathrm{n}_{1}} \mathcal{Y}_{3}^{l_{0}-\mathrm{n}_{1}-\mathrm{n}_{2}} \mathcal{H}_{1}^{\mathrm{n}_{1}} \mathcal{H}_{2}^{\mathrm{n}_{2}} \mathcal{H}_{0}^{\mathrm{n}_{0}} \mathcal{T}^{1}\left(X_{1}, W_{1}\right) \\
& \times\left.\mathcal{T}^{2}\left(X_{2}, W_{2}\right) \mathcal{T}^{0}\left(X_{0}, W_{0}\right)\right|_{X_{r}=X(\lambda)}, \\
= & \left(\eta^{A_{1} B_{1}} \ldots \eta^{A_{\mathrm{n}_{0}} B_{\mathrm{n}_{0}}}\right)\left(\eta^{A_{\mathrm{n}_{0}+1} C_{1}} \ldots \eta^{A_{\mathrm{n}_{0}+\mathrm{n}_{2}} C_{\mathrm{n}_{2}}}\right)\left(\eta^{B_{\mathrm{n}_{0}+1} C_{\mathrm{n}_{2}+1}} \ldots \eta^{\left.B_{\mathrm{n}_{0}+\mathrm{n}_{1}} C_{\mathrm{n}_{2}+\mathrm{n}_{1}}\right)}\right. \\
& \times\left[\frac{\partial}{\partial X}\right]^{\left(C_{\mathrm{n}_{1}+\mathrm{n}_{2}+1} \ldots C_{l_{0}}\right)} \mathcal{T}_{\left\{A_{1} \ldots A_{l_{1}}\right\}}^{1}(X) \mathcal{T}_{\left\{B_{1} \ldots B_{l_{2}}\right\}}^{2}(X) \\
& \times\left.\left[\frac{\partial}{\partial X}\right]^{\left(A_{\mathrm{n}_{2}+\mathrm{n}_{0}+1} \ldots A_{l_{1}}\right)\left(B_{\mathrm{n}_{1}+\mathrm{n}_{0}+1} \ldots B_{l_{2}}\right)} \mathcal{T}_{\left\{C_{1} \ldots C_{l_{0}}\right\}}^{0}(X)\right|_{X=X(\lambda)},
\end{aligned}
$$

where $\mathcal{T}_{\left\{A_{1} \ldots A_{\left.l_{r}\right\}}\right\}}^{r}(X)$ is a STT embedding space tensor field which is projected to symmetric traceless tensor field in $\mathrm{AdS}_{d+1}$ and various differential operators are defined to be:

$$
\begin{array}{lll}
\mathcal{Y}_{1}=\partial_{W_{1}} \cdot \partial_{X_{0}}, & \mathcal{Y}_{2}=\partial_{W_{2}} \cdot \partial_{X_{0}}, & \mathcal{Y}_{3}=\partial_{W_{0}} \cdot \partial_{X_{1}}, \\
\mathcal{H}_{1}=\partial_{W_{2}} \cdot \partial_{W_{0}}, & \mathcal{H}_{2}=\partial_{W_{0}} \cdot \partial_{W_{1}}, & \mathcal{H}_{0}=\partial_{W_{1}} \cdot \partial_{W_{2}} .
\end{array}
$$

\footnotetext{
${ }^{10}$ Notice that while $\left\{\mathrm{n}_{1}, \mathrm{n}_{2}, \mathrm{n}_{0}\right\}$ satisfy the same conditions $\left\{n_{10}, n_{20}, n_{12}\right\}$ (3.2), as we will see from explicit computation, they are not directly identified with each other in obvious manner.
} 
Here we have almost adopted the general parameterizations found in $[12,13]$ with an essential modification on the choice of operator $\mathcal{Y}_{1}$, which is changed from $\partial_{W_{1}} \cdot \partial_{X_{2}} \rightarrow \partial_{W_{1}} \cdot \partial_{X_{0}}$, we shall now explain the need for this modification. Notice that in original parameterization, which integrates over the entire AdS space, such a change is equivalent up to equation of motion and a boundary term which we can safely discard. However restricting along the geodesic $\gamma_{12}$, we have made an explicit choice of external legs, i.e. the curves connecting $X(\lambda)$ and $P_{1,2}$ and internal leg connecting $X(\lambda)$ and $P_{0}$ which will be joined to form four point geodesic Witten diagram as in section 2 , such a cyclic symmetry permuting the three tensor fields is explicitly broken. If we use the original parameterization, certain tensor structures appearing in the corresponding CFT three point function become missing.

Let us workout a simple example of spin-scalar-scalar $(l, 0,0)$ case to illustrate this. First we consider the parameterization used in $[12,13]$

$$
\mathcal{I}_{l, 0,0}^{0,0,0}=\left.\left(\partial_{W_{1}} \cdot \partial_{X_{2}}\right)^{l} \mathcal{T}_{1}\left(X_{1}, W_{1}\right) \mathcal{T}_{2}\left(X_{2}, W_{2}\right) \mathcal{T}_{0}\left(X_{0}, W_{0}\right)\right|_{X_{r}=X},
$$

and when we apply this vertex to integrate over the entire AdS-space, we have:

$$
\begin{aligned}
& \int_{\mathrm{AdS}} d X \frac{\left(2 P_{2} \cdot \mathrm{C}_{1} \cdot X\right)^{l}}{\left(-2 P_{1} \cdot X\right)^{\Delta_{1}+l}} \frac{1}{\left(-2 P_{2} \cdot X\right)^{\Delta_{2}+l}} \frac{1}{\left(-2 P_{0} \cdot X\right)^{\Delta_{0}}} \\
& \propto\left(P_{2} \cdot D_{P_{1}}\right)^{l} \mathcal{A}_{3}^{\Delta_{1}, \Delta_{2}, \Delta_{0}}\left(P_{1}, P_{2}, P_{0}\right) \propto\left[\mathrm{V}_{1,20}\right]^{l} \mathcal{A}_{3}^{\Delta_{1}+l, \Delta_{2}, \Delta_{0}}\left(P_{1}, P_{2}, P_{0}\right) .
\end{aligned}
$$

Here $D_{P_{i}}^{A}$ is given by:

$$
D_{P_{i}}^{A}=Z_{i}^{A}\left(Z_{i} \cdot \frac{\partial}{\partial Z_{i}}-P_{i} \cdot \frac{\partial}{\partial P_{i}}\right)+P_{i}^{A}\left(Z_{i} \cdot \frac{\partial}{\partial P_{i}}\right)
$$

and $\mathcal{A}_{3}^{\Delta_{1}, \Delta_{2}, \Delta_{0}}$ is given by the scalar integral (B.2) in the appendix. This vertex (4.7) precisely reproduces the only and correct corresponding tensor structure in CFT side as we expected. However if we use the same interaction vertex as before but now restricted along geodesic $\gamma_{12}$ :

$$
\mathcal{I}_{l, 0,0}^{0,0,0}=\left.\left(\partial_{W_{1}} \cdot \partial_{X_{2}}\right)^{l} \mathcal{T}_{1}\left(X_{1}, W_{1}\right) \mathcal{T}_{2}\left(X_{2}, W_{2}\right) \mathcal{T}_{0}\left(X_{0}, W_{0}\right)\right|_{X_{r}=X(\lambda)},
$$

we now have

$$
\int_{-\infty}^{+\infty} d \lambda \frac{\left(2 P_{2} \cdot \mathrm{C}_{1} \cdot X(\lambda)\right)^{l}}{\left(-2 P_{1} \cdot X(\lambda)\right)^{\Delta_{1}+l}} \frac{1}{\left(-2 P_{2} \cdot X(\lambda)\right)^{\Delta_{2}+l}} \frac{1}{\left(-2 P_{0} \cdot X(\lambda)\right)^{\Delta_{0}}}=0
$$

due to the accidental orthogonality condition $2 P_{2} \cdot \mathrm{C}_{1} \cdot X(\lambda)=0$ which only occurs along $\gamma_{12} \cdot{ }^{11}$ Now if use the new parametrization given in (4.4) instead, again we only have one type of interaction given by:

$$
\mathcal{J}_{l, 0,0}^{0,0,0}=\left.\left(\partial_{W_{1}} \cdot \partial_{X_{0}}\right)^{l} \mathcal{T}_{1}\left(X_{1}, W_{1}\right) \mathcal{T}_{2}\left(X_{2}, W_{2}\right) \mathcal{T}_{0}\left(X_{0}, W_{0}\right)\right|_{X_{r}=X(\lambda)} .
$$

The corresponding computation along the geodesic $\gamma_{12}$ is given by (up overall constant):

$$
\int_{-\infty}^{+\infty} d \lambda \frac{\left(2 P_{0} \cdot \mathrm{C}_{1} \cdot X(\lambda)\right)^{l}}{\left(-2 P_{1} \cdot X(\lambda)\right)^{\Delta_{1}+l}} \frac{1}{\left(-2 P_{2} \cdot X(\lambda)\right)^{\Delta_{2}}} \frac{1}{\left(-2 P_{0} \cdot X(\lambda)\right)^{\Delta_{0}+l}} \propto\left[\mathrm{V}_{1,20}\right]^{l} \mathcal{A}_{3}^{\Delta_{1}+l \Delta_{2} \Delta_{0}}
$$

\footnotetext{
${ }^{11}$ Similar cancelation was also noted in the recent preprint [16].
} 
where we have used $2 P_{0} \cdot \mathrm{C}_{1} \cdot X(\lambda)=-e^{-\lambda} \sqrt{-2 P_{1} \cdot P_{2}} \mathrm{~V}_{1,02}$. We have now seen that the modified parameterization instead gives the desired CFT tensor structure.

We shall adopt the minimally modified parameterization (4.2) in our computation of the three point geodesic Witten diagrams for symmetric traceless tensor fields. One important feature here is that for given $\left(l_{1}, l_{2}, l_{0}\right)$, the allowed range of the non-negative integers $\left\{\mathrm{n}_{1}, \mathrm{n}_{2}, \mathrm{n}_{0}\right\}$ imply that we have the same number (3.6) of independent interaction vertices as the independent box tensor structures given in (3.3), this implies that we should be able to express the resultant three point GWDs as linear combinations of these box tensor structures, echoing our general argument in the beginning of this section. Moreover as shown in [13], the three point Witten diagrams produced by the original parameterization of three point vertices can also be expressed in terms of the same set of box tensor structures, this implies that we should also be able to expand the ordinary three point Witten diagrams in terms of three point GWDs. We will explicitly do so in a example that follows. One further remark is that the we have chosen $\mathcal{Y}_{3}=\partial_{W_{0}} \cdot \partial_{X_{1}}$ in (4.2), the possible choice is $\mathcal{Y}_{3}=\partial_{W_{0}} \cdot \partial_{X_{2}}$. But this choice is equivalent to starting with cyclically permuted three point vertices in [13], then make similar modification of the differential operator to switch the partial derivative to act on $X_{0}$. We believe for this other choice and the story should go through the same.

\subsection{The $\left(l_{1}, l_{2}, 0\right)$ case}

Let us first consider the case with two external symmetric tensor fields with spins $l_{1,2}$ and one internal scalar field. We have the counting:

$$
l_{0}=0, \quad l_{1}-\mathrm{n}_{0} \geq 0, \quad l_{2}-\mathrm{n}_{0} \geq 0, \quad \mathrm{n}_{1}=\mathrm{n}_{2}=0
$$

The corresponding interaction vertices in this case are:

$$
\begin{aligned}
\mathcal{J}_{l_{1}, l_{2}, 0}^{0,0, \mathrm{n}_{0}}= & \left(\partial_{W_{1}} \cdot \partial_{X_{0}}\right)^{l_{1}-\mathrm{n}_{0}}\left(\partial_{W_{2}} \cdot \partial_{X_{0}}\right)^{l_{2}-\mathrm{n}_{0}}\left(\partial_{W_{1}} \cdot \partial_{W_{2}}\right)^{\mathrm{n}_{0}} \mathcal{T}^{1}\left(X_{1}, W_{1}\right) \\
& \times\left.\mathcal{T}^{2}\left(X_{2}, W_{2}\right) \mathcal{T}^{0}\left(X_{0}, W_{0}\right)\right|_{X_{r}=X(\lambda)}
\end{aligned}
$$

which yield the following integral:

$$
\begin{aligned}
& \mathbb{C} \int_{\gamma_{12}} \eta^{A_{1} B_{1}} \ldots \eta^{A_{n_{0}} B_{n_{0}}} \frac{\left(2 X \cdot \mathrm{C}_{1}\right)_{A_{1} \ldots A_{l_{1}}} \frac{\left(2 X \cdot \mathrm{C}_{2}\right)_{B_{1} \ldots B_{l_{2}}}}{\left(-2 P_{1} \cdot X\right)^{\tau_{1}}}}{\times\left(\frac{\partial}{\partial X}\right)^{A_{n_{0}+1} \ldots A_{l_{1}}}\left(\frac{\partial}{\partial X}\right)^{B_{n_{0}+1} \ldots B_{l_{2}}} \frac{1}{\left(-2 P_{0} \cdot X\right)^{\tau_{2}} \Delta_{0}}} \\
& =\mathbb{C} 2^{l_{1}+l_{2}-2 n_{0}}(-1)^{l_{1}-n_{0}} \beta_{\tau_{12}, \Delta_{0}}\left(\frac{\tau_{12}+\Delta_{0}}{2}\right)_{l_{2}-n_{0}}\left(\frac{\Delta_{0}-\tau_{12}}{2}\right)_{l_{1}-n_{0}}\left[\begin{array}{ccc}
\Delta_{1} & \Delta_{2} \Delta_{0} \\
l_{1} & l_{2} & 0 \\
0 & 0 & n_{0}
\end{array}\right]
\end{aligned}
$$

where $\mathbb{C}=\prod_{r=1}^{3} \mathcal{C}_{\Delta_{r}, l_{r}}$. In this case, happily we found exact one box tensor structure for each interaction vertex. 


\subsection{The $(1,1,2)$ case}

In the most general case involving three symmetric traceless fields with spins $l_{1,2}$ and $l_{0}$, as noted in $[12,13]$, the corresponding three point ordinary Witten diagrams can only be expressed in terms of linear combination of box tensor basis (3.3). The same thing happens for the geodesic vertices in (4.2) and the resultant three point geodesic Witten diagrams, they can only be expressed in terms of linear combination of box basis.

As an illustrative example, we consider the case where $\left(l_{1}, l_{2}, l_{0}\right)=(1,1,2)$. First from the corresponding CFT three point correlation function, we expect there are five box tensor structures arising, they are:

$$
\begin{array}{lll}
{\left[I_{1}\right]:=\left[\begin{array}{ccc}
\Delta_{1} & \Delta_{2} & \Delta_{0} \\
1 & 1 & 2 \\
0 & 0 & 0
\end{array}\right],} & {\left[I_{2}\right]:=\left[\begin{array}{ccc}
\Delta_{1} & \Delta_{2} & \Delta_{0} \\
1 & 1 & 2 \\
1 & 0 & 0
\end{array}\right],} & {\left[I_{3}\right]:=\left[\begin{array}{ccc}
\Delta_{1} & \Delta_{2} & \Delta_{0} \\
1 & 1 & 2 \\
0 & 1 & 0
\end{array}\right],} \\
{\left[I_{4}\right]:=\left[\begin{array}{ccc}
\Delta_{1} & \Delta_{2} & \Delta_{0} \\
1 & 1 & 2 \\
1 & 1 & 0
\end{array}\right],} & {\left[I_{5}\right]:=\left[\begin{array}{ccc}
\Delta_{1} & \Delta_{2} & \Delta_{0} \\
1 & 1 & 2 \\
0 & 0 & 1
\end{array}\right] .}
\end{array}
$$

From the vertex parameterization (4.2), we now also have five independent interaction vertices. Let us denote the integral for the resultant three point geodesic Witten diagram for each vertex by $\left[J_{1,1,2}^{\mathrm{n}_{1}, \mathrm{n}_{2}, \mathrm{n}_{0}}\right]$. The order of $\left\{\mathrm{n}_{1}, \mathrm{n}_{2}, \mathrm{n}_{0}\right\}$ we pick is

$$
\left[J_{1}\right]:=\left[J_{1,1,2}^{0,0,0}\right],\left[J_{2}\right]:=\left[J_{1,1,2}^{1,0,0}\right],\left[J_{3}\right]:=\left[J_{1,1,2}^{0,1,0}\right],\left[J_{4}\right]:=\left[J_{1,1,2}^{1,1,0}\right],\left[J_{5}\right]:=\left[J_{1,1,2}^{0,0,1}\right] .
$$

The actual calculations producing them are complicated but somehow mechanical, however we can keep using the recursive relations of for the anti-symmetric tensor $\mathrm{C}_{i A B}$ listed in appendix $\mathrm{D}$ to show that they can all be expressed in terms of box tensor structures given in (4.17).

We can express the final results through the following matrix multiplication: $\left[J_{a}\right]=$ $\mathbb{T}_{a b}\left[I_{b}\right], a, b=1, \ldots, 5$ where the mixing matrices $\mathbb{T}_{a b}$ for simplified case $\Delta_{2}=\Delta_{1}, \Delta_{0}=\Delta$ is given by:

$$
\begin{gathered}
\mathbb{T}_{a b}=4\left(1+\Delta_{1}\right) \beta_{0, \Delta+2} \mathbb{C} \\
\left(\begin{array}{ccccc}
-\left(-4+\Delta^{2}\right)\left(2+\Delta_{1}\right) & \frac{2(2+\Delta)\left(1+\Delta+\Delta_{1}\right)}{\Delta} & 2(2+\Delta)\left(2+\Delta_{1}\right) & \frac{2(2+\Delta)\left(1+\Delta+\Delta_{1}\right)}{\Delta} & 0 \\
-\Delta & -1-\Delta & -\frac{\Delta+\Delta^{2}+2 \Delta_{1}}{\Delta+\Delta \Delta_{1}} & -\frac{(1+\Delta)\left(\Delta+\Delta_{1}\right)}{\Delta\left(1+\Delta_{1}\right)} & 0 \\
-2+\Delta & -2 & -1-\frac{2}{\Delta}+\Delta & -\frac{1+\Delta}{\Delta} & 0 \\
\frac{1}{1+\Delta_{1}} & \frac{1+\Delta}{\Delta+\Delta \Delta_{1}} & \frac{1+\Delta}{\Delta+\Delta \Delta_{1}} & \frac{1+\Delta}{\Delta+\Delta \Delta_{1}} & 0 \\
0 & 0 & 0 & 0 & \Delta_{1}
\end{array}\right) .
\end{gathered}
$$

In particular, one can check that $\mathbb{T}$ is invertible such that:

$$
\operatorname{Det}\left[\mathbb{T}_{a b}\right] \propto \frac{(-1+\Delta)^{3}(2+\Delta)^{2} \Delta_{1}^{2}\left(1+\Delta_{1}\right)^{3}\left(2(1+\Delta)^{2}+\left(2+2 \Delta+\Delta^{2}\right) \Delta_{1}\right)}{\Delta^{3}} \neq 0,
$$

This implies that we can equivalently express each three point function tensor structures listed in (4.17) in terms of linear combination of three point GWDs for various vertices 
in (4.18). This clearly illustrate that, the holographic dual of three point function for primary operators with spins, as expressed in the box tensor basis, generally requires more than one type of interaction vertices, and to find the ideal basis for two sets of quantities which give one to one correspondence, this essentially becomes a matrix diagonalization problem. ${ }^{12}$ Moreover, recalling that we further can connect the box tensor basis appearing in (4.17) with their corresponding differential tensor basis (3.13):

$$
\begin{array}{ll}
\left\{D_{1}\right\}:=\left\{\begin{array}{ccc}
\Delta_{1} & \Delta_{2} & \Delta_{0} \\
1 & 1 & 2 \\
0 & 0 & 0
\end{array}\right\}, \quad\left\{D_{2}\right\}:=\left\{\begin{array}{ccc}
\Delta_{1} & \Delta_{2} & \Delta_{0} \\
1 & 1 & 2 \\
1 & 0 & 0
\end{array}\right\}, \quad\left\{D_{3}\right\}:=\left\{\begin{array}{ccc}
\Delta_{1} & \Delta_{2} & \Delta_{0} \\
1 & 1 & 2 \\
0 & 1 & 0
\end{array}\right\}, \\
\left\{D_{4}\right\}:=\left\{\begin{array}{ccc}
\Delta_{1} & \Delta_{2} & \Delta_{0} \\
1 & 1 & 2 \\
1 & 1 & 0
\end{array}\right\}, \quad\left\{D_{5}\right\}:=\left\{\begin{array}{ccc}
\Delta_{1} & \Delta_{2} & \Delta_{0} \\
1 & 1 & 2 \\
0 & 0 & 1
\end{array}\right\} .
\end{array}
$$

Again for $\Delta_{1}=\Delta_{2}$ and $\Delta_{0}=\Delta$, their mixing matrix is given by:

$$
\mathbb{A}_{a b}=\left(\begin{array}{ccccc}
1-\frac{1}{4} \Delta(4+\Delta) & -\frac{\Delta}{2} & -\frac{\Delta}{2} & -\frac{1}{2} & \frac{2-\Delta}{4} \\
-\frac{1}{4}(-2+\Delta) \Delta & \frac{\Delta}{2} & 1-\frac{\Delta}{2} & \frac{1}{2} & -\frac{\Delta}{4} \\
-\frac{1}{4}(-2+\Delta) \Delta & 1-\frac{\Delta}{2} & \frac{\Delta}{2} & \frac{1}{2} & -\frac{\Delta}{4} \\
-\frac{1}{4}(-2+\Delta)^{2} & \frac{1}{2}(-2+\Delta) & \frac{1}{2}(-2+\Delta) & -\frac{1}{2} & \frac{2-\Delta}{4} \\
0 & 0 & 0 & 0 & 1
\end{array}\right)
$$

such that $\left\{D_{a}\right\}=\mathbb{A}_{a b}\left[I_{b}\right]$, one can show that $\mathbb{A}_{a b}^{-1}$ is again invertible and agrees with Example 3.3.3 in [10] for $l=2$. It should now be clear that, through two successive matrix multiplications, we can directly relate the differential tensor basis, which are somewhat more natural for constructing the integral representation of spinning conformal partial waves as explained in the previous section, to the three point GWDs for different interaction vertices. We can succinctly summarize it as:

$$
\left\{D_{a}\right\}=\left(\mathbb{A T}^{-1}\right)_{a b}\left[J_{b}\right]
$$

again it would be very interesting to find the new combination of interaction vertices which diagonalizes the matrix $\mathbb{A T}^{-1}$, such that we can have the simple one to one correspondence with the CFT differential tensor basis.

Comments on gluing procedure. So far, we have considered three point geodesic diagrams with a certain interaction. Here we assume generic three point GWDs with external spins $\left(l_{1}, l_{2}, J\right)$ and an arbitrary interaction. To use the gluing identity (2.35), the dimension $\Delta_{0}$ is taken as $h+i \nu .{ }^{13}$ After the geodesic integration, the resultant three point GWD is written in terms of the box tensor structures, and we can reproduce the same box

\footnotetext{
${ }^{12}$ Here we should however mention here that in recent preprint [17], using the new CFT tensor basis constructed from linear combination of (3.4) and (3.5), and suitably constructed AdS space differential operators, the progress for direct identifications between CFT tensor structures and AdS interaction vertices has been made.

${ }^{13}$ For the right side diagram, it is taken as $h-i \nu$.
} 
tensor structure using a summation of the differential operators as in (3.13). Therefore we can write the following relation;

$$
\mathcal{D}_{\text {Left }}^{\left(l_{1}, l_{2}, J\right)} I_{\mathrm{GWD}}^{(0,0, J)}=\text { (coeff.) } I_{\mathrm{GWD}}^{\left(l_{1}, l_{2}, J\right)},
$$

where $I_{\mathrm{GWD}}^{(0,0, J)}$ is the three point GWD with $(0,0, J)$ external spins which computed in section 2 and $\mathcal{D}_{\text {Left }}^{\left(l_{1}, l_{2}, J\right)}$ is a linear summation of operators $\mathcal{D}_{\text {Left }}^{n_{10}, n_{20}, n_{12}}$ defined in (3.16) which produces the same tensor structures as $I_{\mathrm{GWD}}^{\left(l_{1}, l_{2}, J\right)}$. The coefficient in r.h.s., denoted as (coeff.) comes from the action of $\mathcal{D}_{\text {Left }}$. $\mathcal{D}_{\text {Left }}$ produces only the Pochhammer symbols involving $\nu$ which do not give any additional poles when performing the $\nu$ integration. For $I_{\mathrm{GWD}}^{(0,0, J)}$, we already know how these two geodesic diagrams can be glued together in section 2, cf. (2.26). If $\mathcal{D}_{\text {Left }}$ and $\mathcal{D}_{\text {Right }}$ act on the both side of (2.35), in the r.h.s., we obtain the same differential basis as in (4.24). On the other hands, the l.h.s. becomes the corresponding spinning conformal partial wave. In this way, we can concern the gluing process for an arbitrary pair of three point GWDs.

Having illustrated how the three point interaction vertices parameterized in (4.2) can be expressed in terms of the linear combination of box tensor basis, we can summarize the general strategy for constructing four point spinning GWDs which are holographic dual to the spinning conformal partial wave listed in (3.15) as follows:

1. First consider a pair of triplets of CFT primary operators with scaling dimensions and spins $\left(\Delta_{1,2}, l_{1,2}\right)$ and $(h+i \nu, J)$ and $\left(l_{3,4}, \Delta_{3,4}\right)$ and $(h-i \nu, J)$, compute all the resultant three point spinning GWDs for a given pair of vertices parameterized (4.2), and express them in terms of the linear combination box tensor basis, i.e. working out the $\mathbb{T}$-matrix.

2. For each box tensor basis appearing, we further rewrite them into corresponding differential tensor basis, i.e. working out the $\mathbb{A}$ matrix.

3. We can next fuse the resultant differential basis together to obtain the direct relation between the four point spinning GWDs constructed from this pair of three point vertices and the spinning conformal partial waves.

4. Finally, if we consider all possible pairs of interaction vertices for the operators involved, and repeat the steps $1,2,3$, we can then invert the relation between the spinning GWDs and spinning conformal partial waves, and express the spinning conformal partial waves in terms of linear combination of spinning GWDs instead.

\section{Decomposition of Witten diagrams via split representation}

In this section, we discuss how to decompose both four point scalar and spinning Witten diagrams involving general spin- $J$ exchange into four point geodesic Witten diagrams for the single and double trace operators. The original analysis of decomposition have been done in [1] for $J=0,1$ exchanges, the analysis we perform here rely on the so-called "split representation" of the bulk to bulk propagator introduced in [14], and this makes 
clear why we can naturally construct various four point geodesic Witten diagrams from the three point ones, and their connection with the integral representation of conformal block itself. One can regard the cutting identity (2.19) which was used in the previous sections as the natural consequence of the split representation.

We should clarify here that the analysis in this section can be regarded as a recasting the conformal partial wave decompositions of the four point ordinary scalar Witten diagrams done in $[14,15,27]$ directly in terms of geodesic Witten diagrams. To do so we precisely identify the three point GWD contributions in the resultant split representation, while the remaining factors determine the spectrum of exchanged operators, the computational details can be found in appendix E. We will see this somewhat easier approach, which is different from the one used in [1], directly leads to the decomposition of ordinary Witten diagrams into GWDs for arbitrary spin $J$ and it is easier to generalize to the Witten diagrams for external operators with spins. ${ }^{14}$

It was shown in [14] the bulk to bulk propagator (2.10) in so-called traceless gauge, ${ }^{15}$ can be expressed as:

$$
\begin{aligned}
\Pi_{\Delta, J}(X, \tilde{X} ; W, \tilde{W}) & =\sum_{l=0}^{J} \int_{-\infty}^{\infty} d \nu a_{l}(\nu)(W \cdot \nabla)^{J-l}(\tilde{W} \cdot \tilde{\nabla})^{J-l} \Omega_{\nu, l}(X, \tilde{X} ; W, \tilde{W}) . \\
\Omega_{\nu, l}(X, \tilde{X} ; W, \tilde{W}) & =\frac{\nu^{2}}{\pi l !(h-1)_{l}} \int_{\partial} d P_{0} \Pi_{h+i \nu, l}\left(X, P_{0} ; W, D_{Z_{0}}\right) \Pi_{h-i v, l}\left(\tilde{X}, P_{0} ; \tilde{W}, Z_{0}\right) .
\end{aligned}
$$

Here the embedding space covariant derivative $\nabla_{A}$ (or $\tilde{\nabla}_{\tilde{A}}$ ) is defined in (A.19), and it satisfies properties $X^{A} \nabla_{A}=0$ and $\nabla_{A} G_{B C}=0$. The function $\Omega_{\nu, l}(X, \tilde{X} ; W, \tilde{W})$ is the spin- $l$ harmonic in $A_{d} S_{d+1}$ space, $P_{0}$ and $Z_{0}$ denote the coordinate of the boundary point to be integrated over and its auxiliary polarization vector. The key feature of the representation here is that we have expressed the AdS-harmonic functions in terms of the products of the bulk to boundary propagator $\Pi_{h \pm i \nu, l}\left(X, P_{0} ; W, Z_{0}\right)$, hence the name "split representation". Here the meromorphic functions $a_{l}(\nu), l=0,1, \ldots, J$ have been obtained in [14] by comparing with the spectral functions in the conformal partial wave expansion of the corresponding CFT four point correlation function:

$$
\begin{aligned}
a_{J}(\nu) & =\frac{1}{\nu^{2}+(\Delta-h)^{2}}, \\
a_{l}(\nu) & =\sum_{q=1}^{J-l} \frac{(l+q) !}{l ! q !} \frac{(-1)^{q+1}}{2^{q-1}(q-1) !(h+l)_{q-1}} \frac{a_{l+q}(i(h-1+l))}{\nu^{2}+(h+l+q-1)^{2}} .
\end{aligned}
$$

It is interesting to note that only $a_{J}(\nu)$ contains simple poles whose locations explicitly depend on scale dimension $\Delta$, while $a_{l}(\nu)$ for $l<J$ are determined recursively by demanding the cancelation of the residues for spurious poles in CFT spectral functions.

In the following, we will demonstrate how spin- $J$ exchange diagrams with scalar external fields are decomposed into conformal partial waves/geodesic Witten diagrams. The

\footnotetext{
${ }^{14}$ We are grateful to Charlotte Sleight, whose comments encouraged us to explain our intentions better.

${ }^{15}$ One should note that bulk-bulk propagator for spin- $J$ tensor field can also be expressed in other gauge choice [15].
} 

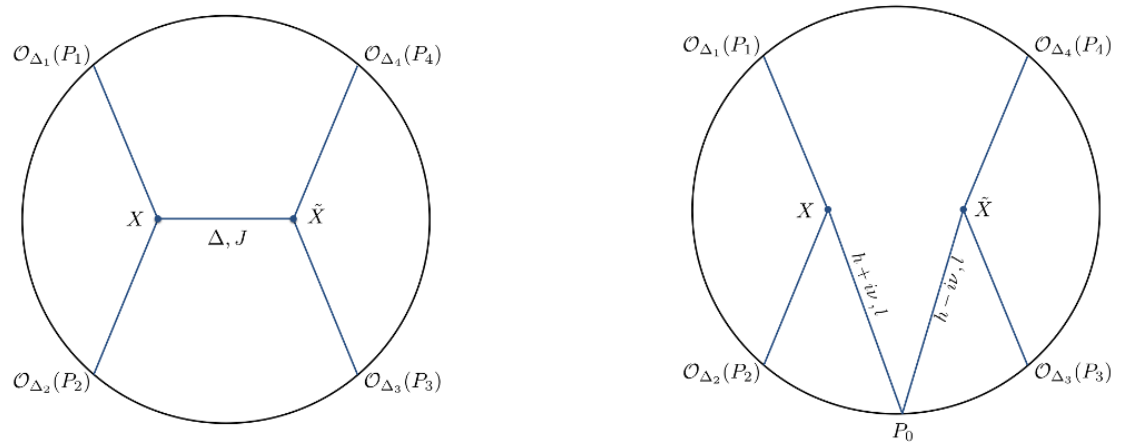

Figure 4. Left: normal exchange Witten diagram with four scalar external fields and a spin- $J$ internal field, Right: a split diagram.

spin- $J$ operator exchange Witten diagram with 4 external scalar fields is given (see the left diagram in figure 4):

$$
\begin{aligned}
W_{(0,0), J,(0,0)}^{4 \text {-pt. }} \equiv & \frac{1}{\left(J !\left(\frac{d-1}{2}\right)_{J}\right)^{2}} \int d X d \tilde{X} \frac{1}{\left(-2 P_{1} \cdot X\right)^{\Delta_{1}}}\left(K \cdot \nabla_{X}\right)^{J} \frac{1}{\left(-2 P_{2} \cdot X\right)^{\Delta_{2}}} \\
& \times \frac{1}{\left(-2 P_{3} \cdot \tilde{X}\right)^{\Delta_{3}}}\left(\tilde{K} \cdot \nabla_{\tilde{X}}\right)^{J} \frac{1}{\left(-2 P_{4} \cdot \tilde{X}\right)^{\Delta_{4}}} \Pi_{\Delta, J}(X, \tilde{X} ; W, \tilde{W}) .
\end{aligned}
$$

Here we dropped unimportant normalization factors $\mathcal{C}_{\Delta_{i}, 0}$. This diagram can be decomposed into product of three point Witten diagrams by using the split representation (5.1) for the bulk to bulk propagator $\Pi_{\Delta, J}(X, \tilde{X} ; W, \tilde{W})$ (see the right digram in figure 4):

$$
\begin{aligned}
& W_{(0,0), J,(0,0)}^{4 \text {-pt. }}=\sum_{l=0}^{J} \int_{\partial} d P_{0} \int_{-\infty}^{\infty} d \nu \frac{\nu^{2}}{\pi} \frac{1}{l !(h-1) !} a_{l}(\nu) \\
& \times\left[\frac{\mathcal{C}_{h+i \nu, l}}{J !\left(\frac{d-1}{2}\right)_{J}} \int d X \frac{1}{\left(-2 P_{1} \cdot X\right)^{\Delta_{1}}}\left(K \cdot \nabla_{X}\right)^{J} \frac{1}{\left(-2 P_{2} \cdot X\right)^{\Delta_{2}}}\left(W \cdot \nabla_{X}\right)^{J-l} \frac{\left(V_{0}\left(X, D_{Z_{0}}\right) \cdot W\right)^{l}}{\left(-2 P_{0} \cdot X\right)^{h+i \nu+l}}\right] \\
& \times\left[\frac{\mathcal{C}_{h-i \nu, l}}{J !\left(\frac{d-1}{2}\right)_{J}} \int d \tilde{X} \frac{1}{\left(-2 P_{3} \cdot \tilde{X}\right)^{\Delta_{3}}}\left(\tilde{K} \cdot \nabla_{\tilde{X}}\right)^{J} \frac{1}{\left(-2 P_{4} \cdot \tilde{X}\right)^{\Delta_{4}}}\left(\tilde{W} \cdot \nabla_{\tilde{X}}\right)^{J-l} \frac{\left(V_{0}\left(\tilde{X}, Z_{0}\right) \cdot \tilde{W}\right)^{l}}{\left(-2 P_{0} \cdot \tilde{X}\right)^{h-i \nu+l}}\right],
\end{aligned}
$$

where we have defined the vector:

$$
V_{0}^{A}(X, Z) \equiv V_{0}^{A}(X)=2\left(X \cdot \mathrm{C}_{0}\right)^{A}=2\left(\left(X \cdot Z_{0}\right) P_{0}^{A}-\left(X \cdot P_{0}\right) Z^{A}\right) .
$$

We will concentrate on three point Witten diagrams in the square parentheses:

$$
\begin{aligned}
& I_{(J, l)}^{\Delta_{1}, \Delta_{2}, h+i \nu} \equiv \\
& \frac{1}{J !\left(\frac{d-1}{2}\right)_{J}} \int_{\text {AdS }} d X \frac{1}{\left(-2 P_{1} \cdot X\right)^{\Delta_{1}}}(K \cdot \nabla)^{J} \frac{1}{\left(-2 P_{2} \cdot X\right)^{\Delta_{2}}}(W \cdot \nabla)^{J-l} \frac{\left(W \cdot V_{0}(X)\right)^{l}}{\left(-2 P_{0} \cdot X\right)^{h+i \nu+l}} .
\end{aligned}
$$


We can simplify the integrands involved as:

$$
\begin{aligned}
& \frac{1}{J !\left(\frac{d-1}{2}\right)_{J}}(K \cdot \nabla)^{J} \frac{1}{\left(-2 P_{2} \cdot X\right)^{\Delta_{2}}}(W \cdot \nabla)^{J-l} \frac{\left(W \cdot V_{0}(X)\right)^{l}}{\left(-2 P_{0} \cdot X\right)^{h+i \nu+l}} \\
& =\sum_{p=0}^{J-l} J_{-l} C_{p}\left(\Delta_{2}\right)_{J}(h+i \nu+l)_{J-l}\left(-2 P_{20}\right)^{J-l-p} \frac{\left(-2 P_{2} \cdot V_{0}(X)\right)^{l}}{\left(-2 P_{2} \cdot X\right)^{\Delta_{2}+J-p}\left(-2 P_{0} \cdot X\right)^{h+i \nu+J-p}}
\end{aligned}
$$

where ${ }_{p} C_{q}=\frac{p !}{(p-q) ! q !}$ is the combinatorial factor in binomial expansion. We can explicitly evaluate the integral (5.8) for these three point Witten diagrams as: ${ }^{16}$

$$
\begin{aligned}
I_{(J, l)}^{\Delta_{1}, \Delta_{2}, h+i \nu}= & \sum_{p=0}^{J-l}{ }_{J-l} C_{p} \frac{(-2)^{J-p}(-1)^{l} \pi^{h}\left(\Delta_{2}\right)_{J}}{\Gamma\left(\Delta_{1}\right) \Gamma\left(\Delta_{2}+J-p\right)}(h+i \nu+J-p)_{p} \Gamma\left(\frac{\Delta_{1}+\Delta_{2}-h \pm i \nu+l}{2}\right) \\
& \times\left(\frac{\Delta_{1}+\Delta_{2}-h+i \nu+l}{2}\right)_{J-l-q}\left(\frac{-\Delta_{12}+h+i \nu+l}{2}\right)_{J-l-q} \\
& \times \beta_{\Delta_{12}, h+i \nu+l}\left[\begin{array}{ccc}
\Delta_{1} & \Delta_{2} & \frac{d}{2}+i \nu \\
0 & 0 & l \\
0 & 0 & 0
\end{array}\right] .
\end{aligned}
$$

To calculate this three point diagram, we used a derivative operator $\mathrm{D}_{02}$ which is defined as

$$
\mathrm{D}_{02} \equiv\left(Z_{0} \cdot P_{2}\right)\left(Z_{0} \cdot \frac{\partial}{\partial Z_{0}}-P_{0} \cdot \frac{\partial}{\partial P_{0}}\right)+\left(P_{0} \cdot P_{2}\right)\left(Z_{0} \cdot \frac{\partial}{\partial P_{0}}\right)
$$

and it satisfies an useful identity:

$$
\left(\mathrm{D}_{02}\right)^{l} \frac{1}{\left(-2 P_{0} \cdot X\right)^{a}}=(a)_{l} \frac{\left(P_{2} \cdot V_{0}(X)\right)^{l}}{\left(-2 P_{0} \cdot X\right)^{a+l}}
$$

In the second equality in (5.10), we used the result in appendix $\mathrm{C}$, and in the last equality in (5.10), note that we used the notation $\Gamma(a \pm b)=\Gamma(a+b) \Gamma(a-b)$ for simplicity. Moreover in the last line we have also isolated the piece which can identified with the integrated results from three point GWD, cf. (2.22) or more generally (B.5). In appendix E, we make this identification more explicit through direct computations.

Now moving to perform the decomposition analysis, we need to consider gluing the product of three point Witten diagrams we just evaluated together by integrating over the spectral parameter $\nu$, the singularity structure of the $\nu$-dependent function multiplying the three point GWD piece crucially determines possible spectrum of the four point GWDs or equivalently scalar conformal blocks can appear. The original four point Witten

\footnotetext{
${ }^{16}$ The same diagram is calculated in [14] by using the series expansion of Gegenbauer polynomials.
} 
diagram (5.5) can be expressed as:

$$
\begin{aligned}
W_{(0,0), J,(0,0)}^{4-\mathrm{pt}}= & \sum_{l=0}^{J} \int_{\partial} d P_{0} \int_{-\infty}^{\infty} d \nu \frac{a_{l}(\nu)}{\pi l !(h-1) !} \sum_{p=0}^{J-l} \sum_{p^{\prime}=0}^{J-l} \Lambda_{\Delta_{1,2,3,4}}^{J, l, p, p^{\prime}}(h \pm i \nu+l)_{J-l} \\
& \left.\times \frac{\mathcal{R}_{\Delta_{1}, \Delta_{2}, h+i \nu}^{J, l, p} \mathcal{R}_{\Delta_{3}, \Delta_{4}, h-i \nu}^{J, l, p^{\prime}}}{(h+i \nu+l)_{J-l-p}(h-i \nu+l)_{J-l-p^{\prime}}}\right) \Gamma\left(\frac{\Delta_{3}+\Delta_{4}-h \pm i \nu+l}{2}\right) \\
& \times \Gamma\left(\frac{\Delta_{1}+\Delta_{2}-h \pm i \nu+l}{2}\right) \\
& \times \mathcal{B}_{h, l}(\nu) \mathcal{K}_{\Delta_{12}, \Delta_{34}, l}(h+i \nu, h-i \nu)\left[\begin{array}{ccc}
\Delta_{1} & \Delta_{2} & h+i \nu \\
0 & 0 & l \\
0 & 0 & 0
\end{array}\right] \cdot\left[\begin{array}{ccc}
\Delta_{3} & \Delta_{4} h-i \nu \\
0 & 0 & l \\
0 & 0 & 0
\end{array}\right] .
\end{aligned}
$$

Let us unpack the various contributions appearing above. Here $\Lambda_{\Delta_{1,2,3,4}}^{J, l, p, p^{\prime}}$ is a factor which does not depend on $\nu$ :

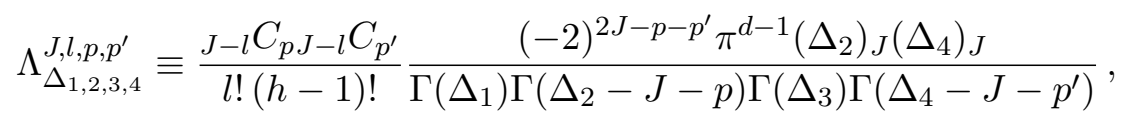

and $\mathcal{R}_{\Delta_{1}, \Delta_{2}, h+i \nu}^{J, l, p}$ is also a regular function of $\nu$ :

$$
\mathcal{R}_{\Delta_{1}, \Delta_{2}, h+i \nu}^{J, p} \equiv\left(\frac{\Delta_{1}+\Delta_{2}-h+i \nu+l}{2}\right)_{J-l-p}\left(\frac{-\Delta_{1}+\Delta_{2}+h+i \nu+l}{2}\right)_{J-l-p} .
$$

$\mathcal{R}_{\Delta_{3}, \Delta_{4}, h-i \nu}^{J l, p^{\prime}}$ is also defined in the similar way. Finally we notice that the last line of (5.13) is almost identical to the integrand appearing in the integral representation of four point scalar GWD (2.35) for spin $l, l=0,1, \ldots, J$, except now we need to carefully examine the pole structures multiplying it. We will again start with the relation (2.30) with $J \rightarrow l$, but now multiply both sides with the following factor and integrate over $\nu$ :

$$
\begin{aligned}
a_{l}(\nu) & (h \pm i \nu+l)_{J-l} \mathcal{R}_{\Delta_{1}, \Delta_{2}, h+i \nu}^{J, l,} \mathcal{R}_{\Delta_{3}, \Delta_{4}, h-i \nu}^{J, l, p^{\prime}} \frac{\mathcal{B}_{h, l}(\nu)}{(h+i \nu+l)_{J-l-p}(h-i \nu+l)_{J-l-p^{\prime}}} \\
& \times \Gamma\left(\frac{\Delta_{1}+\Delta_{2}-h \pm i \nu+l}{2}\right) \Gamma\left(\frac{\Delta_{3}+\Delta_{4}-h \pm i \nu+l}{2}\right) .
\end{aligned}
$$

The l.h.s. is what we have in the summation of (5.13) except for some overall constant factors, the r.h.s. becomes

$$
\begin{aligned}
& \int_{-\infty}^{\infty} d \nu a_{l}(\nu)(h \pm i \nu+l)_{J-l} \frac{\mathcal{R}_{\Delta_{1}, \Delta_{2}, h+i \nu}^{J, l, p} \mathcal{R}_{\Delta_{3}, \Delta_{4}, h-i \nu}^{J, l, p^{\prime}}}{(h+i \nu+l)_{J-l-p}(h-i \nu+l)_{J-l-p^{\prime}}} \\
& \quad \times \frac{1}{2^{l} c_{l}} \Gamma\left(\frac{\Delta_{1}+\Delta_{2}-h \pm i \nu+l}{2}\right) \Gamma\left(\frac{\Delta_{3}+\Delta_{4}-h \pm i \nu+l}{2}\right) \\
& \quad \times\left[\frac{\mathcal{K}_{\Delta_{12}, \Delta_{34}, l}(h+i \nu, h+i \nu)}{(h+i \nu-1)_{l} \Gamma(i \nu) \Gamma(h+i \nu+l)} W_{\mathcal{O}_{h+i \nu, l}}+\frac{\mathcal{K}_{\Delta_{12}, \Delta_{34}, l}(h-i \nu, h-i \nu)}{(h-i \nu-1)_{l} \Gamma(-i \nu) \Gamma(h-i \nu+l)} W_{\tilde{\mathcal{O}}_{h-i \nu, l}}\right] .
\end{aligned}
$$

In the following, we will focus on the integral above and perform the integration over $\nu$ separately for each $l$, since they spectral function $a_{l}(\nu)(5.3)$ differs. 
The highest spin $\boldsymbol{l}=\boldsymbol{J}$ contribution. Here we consider the contribution from the highest spin exchange $l=J$ case in (5.17). Note that in this case, $p$ and $p^{\prime}$ can be taken as only $p=p^{\prime}=0$. Then the integration in (5.17) becomes

$$
\begin{aligned}
& \int_{-\infty}^{\infty} d \nu a_{J}(\nu) \frac{1}{2^{J} c_{J}} \Gamma\left(\frac{\Delta_{1}+\Delta_{2}-h \pm i \nu+J}{2}\right) \Gamma\left(\frac{\Delta_{3}+\Delta_{4}-h \pm i \nu+J}{2}\right) \\
& \times\left[\frac{\mathcal{K}_{\Delta_{12}, \Delta_{34}, J}(h+i \nu, h+i \nu)}{(h+i \nu-1)_{J} \Gamma(i \nu) \Gamma(h+i \nu+J)} W_{\mathcal{O}_{h+i \nu, J}}+\frac{\mathcal{K}_{\Delta_{12}, \Delta_{34}, J}(h-i \nu, h-i \nu)}{(h-i \nu-1)_{J} \Gamma(-i \nu) \Gamma(h-i \nu+J)} W_{\tilde{\mathcal{O}}_{h-i \nu, J}}\right] .
\end{aligned}
$$

As in the calculation in section 2 , the conformal partial wave $W_{\mathcal{O}_{h+i \nu}}$ converges in the lower half plane in the $\nu$ integration, for the first term the integration contour should be taken in the lower half plane. In the second term, the contour is taken in the upper half plane for the same reason. In the first term, $a_{J}(\nu)$ and gamma functions in the first line have the following poles in the lower half plane:

$$
\begin{aligned}
& \nu=-i(\Delta-h), \\
& \nu=-i\left(\Delta_{m, J}^{(12)}-h\right), \quad \nu=-i\left(\Delta_{m, J}^{(34)}-h\right), \quad m=0,1,2, \ldots
\end{aligned}
$$

where the pole in (5.19) comes from the coefficient $a_{J}(\nu)$ and the poles in (5.20) come from gamma functions. Here $\Delta_{m, J}^{(12)}$ is defined as

$$
\Delta_{m, J}^{(12)} \equiv \Delta_{1}+\Delta_{2}+J+2 m
$$

and $\Delta_{m, J}^{(34)}$ is also defined in the similar way. These poles contribute the integration in the first term. Eq. (5.19) corresponds to the contribution from the single trace operator exchange and it is precisely the origin of the integral representation of scalar GWD (2.26). While the remaining poles (5.20) corresponds to the double trace operator exchange. Similarly, in the second term, the following poles contribute;

$$
\begin{aligned}
& \nu=+i(\Delta-h), \\
& \nu=+i\left(\Delta_{m, J}^{(12)}-h\right), \quad \nu=+i\left(\Delta_{m, J}^{(34)}-h\right), \quad m=0,1,2, \ldots
\end{aligned}
$$

Then combining the contribution from the first and second term and multiplying some constant factors to (5.18), we can obtain the following decomposition,

$$
\left.W_{(0,0), J,(0,0)}^{4 \text {-pt. }}\right|_{l=J}=\tilde{\alpha}_{\Delta, J} W_{\mathcal{O}_{\Delta, J}}+\sum_{m=0}^{\infty} \tilde{\alpha}_{\Delta_{m, J}^{(12)}, J} W_{\mathcal{O}_{\Delta_{m, J}^{(12)},}}+\sum_{m=0}^{\infty} \tilde{\alpha}_{\Delta_{m, J}^{(34)}, J} W_{\mathcal{O}_{\Delta_{m, J}^{(34)}}},
$$

where each coefficient is determined by the residue of the corresponding poles. Each conformal partial wave $W_{\mathcal{O}_{\Delta, J}}, W_{\mathcal{O}_{\Delta_{m, J}^{(12), J}}}$ and $W_{\mathcal{O}_{\Delta_{n, J}^{(34), J}}}$ in the r.h.s. is proportional to the corresponding GWDs as in section 2. This expansion leads to the following decomposition into GWD $\mathcal{W}$,

$$
\left.W_{(0,0), J,(0,0)}^{4 \text {-pt. }}\right|_{l=J}=\alpha_{\Delta, J} \mathcal{W}_{\mathcal{O}_{\Delta, J}}+\sum_{m=0}^{\infty} \alpha_{\Delta_{m, J}^{(12), J}} \mathcal{W}_{\mathcal{O}_{\Delta_{m, J}^{(12)},}}+\sum_{m=0}^{\infty} \alpha_{\Delta_{m, J}^{(34)}, J} \mathcal{W}_{\mathcal{O}_{\Delta_{m, J}^{(34)}}}
$$


The spin $l<\boldsymbol{J}$ contributions. Next we consider the $0 \leq l<J$ cases in (5.17). Let us first consider the contributions from the double trace operators, as encoded within the $\Gamma$-functions in the second line of (5.17). In the first term, after the $\nu$-integration, nonvanishing residues arise from the poles at

$$
\nu=-i\left(\Delta_{m, l}^{(12)}-h\right), \quad \nu=-i\left(\Delta_{m, l}^{(34)}-h\right), \quad m=0,1,2, \ldots
$$

and in the second term, the the poles at

$$
\nu=+i\left(\Delta_{m, l}^{(12)}-h\right), \quad \nu=+i\left(\Delta_{m, l}^{(34)}-h\right), \quad m=0,1,2, \ldots
$$

give similar residues. ${ }^{17}$ After integrating over $\nu$ and multiplying some constants, we obtain the following decomposition for the lower $\operatorname{spin} l<J$ case;

$$
\left.W_{(0,0), J,(0,0)}^{4-p t .}\right|_{l<J}=\sum_{m=0}^{\infty} \alpha_{\Delta_{m, l}^{(12)}, l} \mathcal{W}_{\mathcal{O}_{\Delta_{m, l}^{(12)}, l}}+\sum_{m=0}^{\infty} \alpha_{\Delta_{m, l}^{(34),}} \mathcal{W}_{\mathcal{O}_{\Delta_{m, l}^{(34), l}}}
$$

Here the coefficients come from the residues corresponding to each double trace poles.

Together with the result of the highest spin case (5.24) and the lower spin case (5.28), the normal four point exchange diagram with a spin- $J$ internal field can be decomposed as;

$$
W_{(0,0), J,(0,0)}^{4-p t .}=\alpha_{\Delta, J} \mathcal{W}_{\mathcal{O}_{\Delta, J}}+\sum_{l=0}^{J}\left(\sum_{m=0}^{\infty} \alpha_{\Delta_{m, l}^{(12), l}} \mathcal{W}_{\mathcal{O}_{\Delta_{m, l}^{(12)}, l}}+\sum_{m=0}^{\infty} \alpha_{\Delta_{m, l}^{(34), l}} \mathcal{W}_{\mathcal{O}_{\Delta_{m, l}^{(34), l}}}\right) .
$$

However to complete our decomposition analysis here, we notice an essential difference for $l<J$ cases is that there can be so-called "spurious pole contributions" arises [14, 28], from $a_{l<J}(\nu)$ as defined in (5.3). For fixed $l$, they are located at:

$$
\nu= \pm i(h+l+q-1) . \quad q=1, \ldots, J-l
$$

along the imaginary axis in complex $\nu$-plane, such that when we close contour in either lower or upper half plane in (5.17), they give four point GWDs/conformal partial waves associated with integer scaling dimensions which do not depend on $\Delta_{i}$ or $\Delta$. To illustrate these contributions are unphysical, consider the relations (5.1) and (2.15), and the following consistency relation is obtained:

$$
\begin{aligned}
& \Pi_{\Delta, J}(X, \tilde{X} ; W, \tilde{W})= \\
& \sum_{l=0}^{J} \int_{-\infty}^{\infty} d \nu a_{l}(\nu)(W \cdot \nabla)^{J-l}(\tilde{W} \cdot \tilde{\nabla})^{J-l}\left(\Pi_{h+i \nu, l}(X, \tilde{X} ; W, \tilde{W})-\Pi_{h-i \nu, l}(X, \tilde{X} ; W, \tilde{W})\right) .
\end{aligned}
$$

After the $\nu$ integration, from the highest spin term $l=J$, we can obtain the original bulk to bulk propagator, therefore the remaining $l=0,1, \ldots, J-1$ summation which only pick up residues from spurious poles must sum to zero. From this point of view, the spurious pole contributions give no physical contributions. However, when we substitute the split representation (5.1) into the four point Witten diagram, we have performed the $X$ and

\footnotetext{
${ }^{17}$ Note that in fact some of the poles are canceled by zeros of the Pochhammer symbols in (5).
} 
$\tilde{X}$ integration first (or equivalently $\lambda$ and $\lambda^{\prime}$ ), before performing $\nu$-integration, there are additional poles such as the double trace operators poles listed in (5.26) and (5.27) plus regular $\nu$-dependences appearing. In other words, $X$ and $\tilde{X}$ integrations do not commute with $\nu$ integration as should be expected. However crucially for our integrand (5.5), these additional poles do not coincide with the spurious poles or affect convergence of subsequent $\nu$-integration, as far as the final residues arising from the spurious poles are concerned, the $\nu$ integration commutes with $X$ and $\tilde{X}$ integrations. We can thus use the (5.31) to argue that the residues arising from the spurious poles in (5.17), when we sum over all the $l=0, \ldots, J-1$ contributions, should total to zero. This slightly simplified argument is in accord with the recursive relations imposed on $a_{l}(\nu)$ [14], which in turns arise from the cancelation of the spurious residues in the dual Mellin amplitude [28]. This completes our generalization of the decomposition for four point scalar Witten diagrams into four point scalar GWDs done in [1] for $J=0,1$ to arbitrary $J$.

To close this section, we would like to consider possible Mellin representation [29] of scalar GWDs. The Mellin representation of CPWs is already written in [8, 28], which can be identified with its integral representation obtained from two copies of three point functions (2.35) hence the three point GWDs (2.26), explicitly we have the following relation:

$$
\begin{aligned}
& W_{\Delta . J}(u, v) \propto \int_{-\infty}^{\infty} d \nu \frac{\mathcal{B}_{h, J}(\nu)}{\nu^{2}+(\Delta-h)^{2}} \int_{-i \infty}^{i \infty} \frac{d t d s}{(4 \pi i)^{2}} u^{\frac{t}{2}} v^{\frac{-(s+t)}{2}} P_{\nu, J}(s, t)(h \pm i \nu-1)_{J} \\
& \times \Gamma\left(\frac{h \pm i \nu-J-t}{2}\right) \Gamma\left(\frac{t+s}{2}\right) \Gamma\left(\frac{t+s+\Delta_{12}-\Delta_{34}}{2}\right) \Gamma\left(\frac{-\Delta_{12}-s}{2}\right) \Gamma\left(\frac{\Delta_{34}-s}{2}\right), \\
& \propto \int_{-\infty}^{\infty} d \nu \frac{\mathcal{B}_{d, J}(\nu)}{\nu^{2}+(\Delta-h)^{2}} \mathcal{K}_{\Delta_{12}, \Delta_{34} ; J}(h+i \nu, h-i \nu) \int d P_{0}\left[\begin{array}{ccc}
\Delta_{1} \Delta_{2} h+i \nu \\
0 & 0 & J \\
0 & 0 & 0
\end{array}\right] \cdot\left[\begin{array}{ccc}
\Delta_{3} \Delta_{4} & h-i \nu \\
0 & 0 & J \\
0 & 0 & 0
\end{array}\right],
\end{aligned}
$$

where $s, t$ are Mellin integration variables and $P_{\nu, J}(s, t)$ is the Mack polynomial which is defined in the appendix B in [28]. It is known that [30] that Mellin amplitudes exhibits factorization properties when considering the residues associated with the infinite sequence of simple poles located at:

$$
t=h+i \nu-J+2 m, \quad m=0,1,2, \ldots
$$

and also their shadows with $+i \nu \rightarrow-i \nu$. Such that for each spin- $J$ exchange, we can express the residues in terms of the lower point Mellin amplitudes, joined together by certain function which in flat space limit can be identified with the propagator of spin- $J$ particle. In the simplest non-trivial case, we have four point Mellin amplitudes factorized into two copies of three point Mellin amplitudes, joined together by the "propagator". Given the conformal partial waves are building blocks of four point correlation functions, its Mellin representation given in first line of (5.32) inherits such a factorization, and the resultant pieces should be closely related to the building blocks of its holographic counterpart, i.e. three point GWDs, it would be very interesting to clarify such a relation. 
Comments on fields with spins. Here we consider the simple extension of Witten diagrams with external spinning fields. The basic idea is to use the derivative operators defined in (3.7)-(3.10). A simple example can be obtain by using derivative operators $\mathrm{D}_{12}$. If we consider $\mathrm{D}_{12}$ operator acting on the integration (5.8), the following three point diagram appears:

$$
\begin{aligned}
\left(\mathrm{D}_{12}\right)^{l_{1}} I_{b,(J, l)}^{\Delta_{1}, \Delta_{2}, h+i \nu}= & \frac{1}{J !\left(\frac{d-1}{2}\right)_{J} l_{1} !\left(\frac{d-1}{2}\right)_{l_{1}}} \frac{\left(\Delta_{1}\right)_{l_{1}}}{\left(\Delta_{2}\right)_{l_{1}}} \int_{\mathrm{AdS}} d X \frac{\left(\tilde{K} \cdot V_{1}(X)\right)^{l_{1}}}{\left(-2 P_{1} \cdot X\right)^{\Delta_{1}+l_{1}}} \\
& \times(\tilde{W} \cdot \nabla)^{l_{1}}(K \cdot \nabla)^{J} \frac{1}{\left(-2 P_{2} \cdot X\right)^{\Delta_{2}-l_{1}}}(W \cdot \nabla)^{J-l} \frac{\left(W \cdot V_{0}(X)\right)^{l}}{\left(-2 P_{0} \cdot X\right)^{h+i \nu+l}} .
\end{aligned}
$$

This corresponds to $\left(l_{1}, l_{2}, l_{0}\right)=\left(l_{1}, 0, J\right)$ case with an interaction like

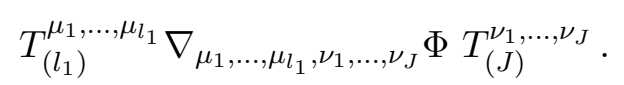

After calculating the integration in (5.34) with $\mathrm{D}_{12}$, the integral is proportional to the following differential basis:

$$
\left\{\begin{array}{ccc}
\Delta_{1} & \Delta_{2} & h+i \nu \\
l_{1} & 0 & l \\
0 & 0 & 0
\end{array}\right\}
$$

as the result of the integration (5.34) should be same as the last line of (5.10) acting with $\left(\mathrm{D}_{12}\right)^{l_{1}}$. The coefficient in front of this basis (5.36) can have $\nu$-dependence through the action of the derivative $\mathrm{D}_{12}$ acting on $\hat{\mathcal{A}}^{\Delta_{1}, \Delta_{2}, h+i \nu}$, however this only resulted in the Pochhammer symbol which does not give additional poles. In this case, we can decompose the spinning Witten diagram with the interaction in (5.35) in a similar way and as in the scalar case, now the conformal partial waves in (5.29) are changed as $\left(\mathrm{D}_{12}\right)^{l_{1}} W_{\mathcal{O}}$ where $W_{\mathcal{O}}$ can be the scalar conformal partial wave for single and double trace operators in the r.h.s. of (5.29).

Even if we consider more general cases with arbitrary external spins and interaction, the 3-point integration can be done basically and the result should be written in terms of the box basis for the same reason as in appendix D. ${ }^{18}$ Then after the same argument, we obtain the single trace and the double trace contribution from the $\nu$ integration. The resulting decomposition is expanded in terms of spinning conformal partial waves like $\mathcal{D}_{\text {left }} \mathcal{D}_{\text {right }} W_{\mathcal{O}}$.

\section{Acknowledgments}

This work was supported in part by National Science Council through the grant 1042112-M-002 -004 -MY, Center for Theoretical Sciences at National Taiwan University. HYC would like to thank Kyoto University, Korea Institute for Advanced Study and Keio University for the opportunities to present part of this work and the hospitalities during its completion. The work of HK is supported by the Japan Society for the Promotion of Science (JSPS) and by the Supporting Program for Interaction-based Initiative Team Studies (SPIRITS) from Kyoto University.

\footnotetext{
${ }^{18} \mathrm{It}$ is difficult to specify the box basis corresponding to an arbitrary interaction. It seem that we should consider on case-by-case basis.
} 


\section{A Embedding formalism}

In this appendix we review the essential details about the embedding space formalism for encoding the tensors in both euclidean $d+1$ dimensional Anti-de Sitter space and the $d$ dimensional euclidean space living on its boundary, this formalism is particularly convenient for studying $\mathrm{AdS}_{d+1} / \mathrm{CFT}_{d}$ correspondence. It is useful to realize the common $\operatorname{SO}(d, 1)$ isometry group of $\mathrm{AdS}_{d+1}$ space and conformal group of its $d$-dimensional boundary as the Lorentz group of a $d+2$ dimensional Minkowski space. The essence of the embedding formalism is that we can realize the non-linear isometry and conformal transformations of the lower dimensional spaces as the linear Lorentz transformation of the associated embedding space, this becomes beneficial when dealing with tensors.

In $d+2$ dimensional embedding space $\mathbb{M}^{d+1,1}$, the euclidean $A d S_{d+1}$ space is defined by the set of future directed unit vectors satisfying:

$$
\operatorname{AdS}_{d+1}: X \cdot X=\eta_{A B} X^{A} X^{B}=-1, \quad \eta_{A B}=\operatorname{diag}(-1,1, \ldots, 1), \quad X^{0}>1
$$

which can also be viewed as a $d+1$ dimensional hyperboloid, and we have set the radius of curvature to be 1 . We can parametrize the solutions to (A.1) explicitly in the light cone coordinates:

$$
\left(X^{+}, X^{-}, X^{a}\right)=\frac{1}{z}\left(1, y^{2}+z^{2}, y^{a}\right), \quad X \cdot X=-X^{+} X^{-}+\delta_{a b} X^{a} X^{b}, \quad a, b=0, \ldots, d-1
$$

in terms of the Poincare coordinates $x^{\mu}=\left(z, y^{a}\right)$ of $\operatorname{AdS}_{d+1}$ space. Towards the boundary $\operatorname{AdS}_{d+1}$, the hyperboloid asymptotes to the light cone $X \cdot X=0$, i.e. the conformal boundary $\mathbb{R}^{d}$ is identified with the projective cone of light rays in the embedding space. They are given by the homogeneous coordinates subjected to the projective identification:

$$
\mathbb{R}^{d}: P \cdot P=0, \quad P^{A} \sim \lambda P^{A}, \quad \lambda \neq 0 .
$$

In terms of Poincare coordinates, the boundary points up to projective identification above are parameterized as:

$$
\left(P^{+}, P^{-}, P^{a}\right)=\left(1, y^{2}, y^{a}\right) .
$$

Next we consider embedding physical tensor fields in $\mathrm{AdS}_{d+1}$ and $\mathbb{R}^{d}$ into embedding space $\mathbb{M}^{d+1,1}$. Explicitly, given an arbitrary rank-r tensor field in $\mathrm{AdS}_{d+1}$ or $\mathbb{R}^{d}$, they are related to their embedding space counterparts through the pull-back operations:

$$
\begin{aligned}
\operatorname{AdS}_{d+1} & : \mathcal{T}_{\mu_{1} \ldots \mu_{r}}^{(\mathrm{AdS})}(x)=\frac{\partial X^{A_{1}}}{\partial x^{\mu_{1}}} \ldots \frac{\partial X^{A_{r}}}{\partial x^{\mu_{r}}} T_{A_{1} \ldots A_{r}}(X), \\
\mathbb{R}^{d}: & \mathcal{F}_{a_{1} \ldots a_{r}}^{(\mathbb{R})}(y)=\frac{\partial P^{A_{1}}}{\partial y^{a_{1}}} \ldots \frac{\partial P^{A_{r}}}{\partial y^{a_{r}}} F_{A_{1} \ldots A_{r}}(P) .
\end{aligned}
$$

In particular, the $\operatorname{AdS}_{d+1}$ and $\mathbb{R}^{d}$ metrics are given by:

$$
\operatorname{AdS}_{d+1}: g_{\mu \nu}^{(\mathrm{AdS})}=\frac{\partial X^{A}}{\partial x^{\mu}} \frac{\partial X^{B}}{\partial x^{\mu}} \eta_{A B}, \quad \mathbb{R}^{d}: \delta_{a b}^{(\mathbb{R})}=\frac{\partial P^{A}}{\partial y^{a}} \frac{\partial P^{B}}{\partial y^{b}} \eta_{A B}
$$


However the pull-back operations defined in (A.5) are surjective but not injective, in other words given a physical tensor in $\operatorname{AdS}_{d+1}$ or $\mathbb{R}^{d}$, they do not have a unique representative in the embedding space $\mathbb{M}^{d+1,1}$, but rather the embedding introduces redundant unphysical degrees of freedom. We can see this from the orthogonal conditions:

$$
\left.X_{A} \frac{\partial X^{A}}{\partial x^{\mu}}\right|_{X \cdot X=-1}=0,\left.\quad P_{A} \frac{\partial P^{A}}{\partial y^{a}}\right|_{P \cdot P=0}=0,
$$

we can see that any tensor components proportional to $X_{\left(A_{1}\right.} H_{\left.A_{2} \ldots A_{r}\right)}(X)$ and $P_{\left(A_{1}\right.} H_{\left.A_{2} \ldots A_{r}\right)}^{\prime}(P)$ contained respectively in $T_{A_{1} \ldots A_{r}}(X)$ and $F_{A_{1} \ldots A_{r}}(P)$ vanish under the pull-back operations in (A.5), hence unphysical. Geometrically we can regard these extra components as being normal to the hypersurface (A.1) and (A.3) respectively. We can thus eliminate these unphysical redundant degrees of freedom in the embedding space tensors by further imposing the transverse condition:

$$
\left.X^{A_{1}} T_{A_{1} \ldots A_{r}}(X)\right|_{X \cdot X=-1}=0,\left.\quad P^{A_{1}} F_{A_{1} \ldots A_{r}}(P)\right|_{P \cdot P=0}=0
$$

such that $T_{A_{1} \ldots A_{r}}(X)$ and $F_{A_{1} \ldots A_{r}}(P)$ only contain the components which are tangent to $\mathrm{AdS}_{d+1}$ and $\mathbb{R}^{d}$ respectively. These are the embedding representatives of the $\operatorname{AdS}_{d+1}$ and $\mathbb{R}^{d}$ tensor fields.

Moreover in the main text, we would like to consider symmetric traceless $\operatorname{AdS}_{d+1}$ and $\mathbb{R}^{d}$ tensor fields. To construct their representatives in embedding space $\mathbb{M}^{d+1,1}$ they need to be symmetric traceless also transverse (STT) from the discussion above, let us first introduce the following generating polynomials:

$$
\begin{aligned}
T(X, W) & =W^{A_{1}} \ldots W^{A_{r}} T_{A_{1} \ldots A_{r}}(X), \quad X \cdot W=W \cdot W=0, \\
F(P, Z) & =Z^{A_{1}} \ldots Z^{A_{r}} F_{A_{1} \ldots A_{r}}(P), \quad P \cdot Z=Z \cdot Z=0 .
\end{aligned}
$$

Here we have introduced the auxiliary vectors $W^{A}$ and $Z^{A}, X \cdot W=0$ and $W \cdot W=0$ imply $T_{A_{1} \ldots A_{r}}(X)$ is defined up to equivalence $\sim X_{\left(A_{1}\right.} H_{\left.A_{2} \ldots A_{r}\right)}(X)+\eta_{\left(A_{1} A_{2}\right.} S_{\left.A_{3} \ldots A_{r}\right)}(X)$, the contraction with $W^{A}$ s only picks up the symmetric, traceless and transverse components. Similarly the properties of the auxiliary vector $P^{A}$ ensures the contraction only picks up the transverse and traceless (plus symmetric) components of $F_{A_{1} \ldots A_{r}}(P)$. It is worth however noting that under the rescaling $F_{A_{1} \ldots A_{r}}(\lambda P)=\lambda^{-\Delta} F_{A_{1} \ldots A_{r}}(P), \lambda>0$, it is a homogenous polynomial of degree $-\Delta$.

To recover embedding space STT tensors representing symmetric traceless $\mathrm{AdS}_{d+1}$ and $\mathbb{R}^{d}$ tensors directly from (A.9) and (A.10), it is convenient to define the operators $K_{A}$ and $D_{A}$ which act on the symmetric products of $W^{A}$ and $Z^{A}$ respectively as:

$$
\begin{aligned}
& \frac{1}{r !\left(\frac{d-1}{2}\right)_{r}} K_{A_{1}} \ldots K_{A_{r}} W^{B_{1}} \ldots W^{B_{r}}=G_{\left\{A_{1}\right.}^{B_{1}} \ldots G_{\left.A_{r}\right\}}^{B_{r}}=G_{\left(A_{1}\right.}^{B_{1}} \ldots G_{\left.A_{r}\right)}^{B_{r}}-\text { traces } \\
& \frac{1}{r !\left(\frac{d-2}{2}\right)_{r}} D_{A_{1}} \ldots D_{A_{r}} Z^{B_{1}} \ldots Z^{B_{r}}=\Pi_{a_{1} \ldots a_{r}}^{b_{1} \ldots b_{r}} \frac{\partial P_{A_{1}}}{\partial y_{a_{1}}} \ldots \frac{\partial P_{A_{r}}}{\partial y_{a_{r}}} \frac{\partial P^{B_{1}}}{\partial y^{b_{1}}} \ldots \frac{\partial P^{B_{r}}}{\partial y^{a_{r}}}
\end{aligned}
$$

where $(.$.$) in the above implies total symmetrization of indices and$

$$
\Pi_{a_{1} \ldots a_{r}}^{b_{1} \ldots b_{r}}=\delta_{\left(a_{1}\right.}^{b_{1}} \ldots \delta_{\left.a_{r}\right)}^{b_{r}}-\text { traces, } \quad \frac{\partial P^{A}}{\partial y^{b}}=\left(0,2 x_{b}, \delta_{b}^{a}\right) .
$$


In other words we obtain the manifestly symmetric, traceless and transverse tensorial projectors, and the resultant embedding space tensors

$$
\begin{aligned}
& T_{\left\{A_{1} \ldots A_{r}\right\}}(X)=G_{\left\{A_{1}\right.}^{B_{1}} \ldots G_{\left.A_{r}\right\}}^{B_{r}} T_{B_{1} \ldots B_{r}}(X) \\
& F_{\left\{A_{1} \ldots A_{r}\right\}}(P)=\Pi_{a_{1} \ldots a_{r}} b_{1} \ldots b_{r} \frac{\partial P_{A_{1}}}{\partial y_{a_{1}}} \ldots \frac{\partial P_{A_{r}}}{\partial y_{a_{r}}} \frac{\partial P^{B_{1}}}{\partial y^{b_{1}}} \ldots \frac{\partial P^{B_{r}}}{\partial y^{a_{r}}} F_{B_{1} \ldots B_{r}}(P)
\end{aligned}
$$

are the desired STT representatives of $\mathrm{AdS}_{d+1}$ and $\mathbb{R}^{d}$ tensors in the embedding space $\mathbb{M}^{d+1,1}$. For completeness, explicit expression for the operators $K_{A}$ and $D_{A}$ can be given in terms of following differential operators:

$$
\begin{aligned}
K_{A}= & \frac{d-1}{2}\left(\frac{\partial}{\partial W^{A}}+X_{A}\left(X \cdot \frac{\partial}{\partial W}\right)\right)+\left(W \cdot \frac{\partial}{\partial W}\right) \frac{\partial}{\partial W^{A}} \\
& +X_{A}\left(W \cdot \frac{\partial}{\partial W}\right)\left(X \cdot \frac{\partial}{\partial W}\right)-\frac{1}{2}\left(\frac{\partial^{2}}{\partial W \cdot \partial W}+\left(X \cdot \frac{\partial}{\partial W}\right)\left(X \cdot \frac{\partial}{\partial W}\right)\right), \\
D_{A}= & \left(\frac{d-2}{2}+Z \cdot \frac{\partial}{\partial Z}\right) \frac{\partial}{\partial Z^{A}}-\frac{1}{2} Z_{A} \frac{\partial^{2}}{\partial Z \cdot \partial Z}
\end{aligned}
$$

however we mostly will not use these somewhat lengthy expressions in the main text, only the formal operations (A.11) and (A.12) will be sufficient. When the contracted embedding space tensor in the generating polynomial is already traceless and transverse, the action of $K_{A}$ simplifies to

$$
K_{A}=\left(\frac{d-1}{2}+W \cdot \frac{\partial}{\partial W}\right) \frac{\partial}{\partial W^{A}}
$$

Finally, we can consider the embedding space representative of $\mathrm{AdS}_{d+1}$ covariant derivative, it acts on the embedding space tensor satisfying the transverse condition (A.8), and the resultant tensor should remain so after its action. The following differential operator in $\mathbb{M}^{d+1,1}$ satisfies such requirement:

$$
\nabla_{A}=\frac{\partial}{\partial X^{A}}+X_{A}\left(X \cdot \frac{\partial}{\partial X}\right)+W_{A}\left(X \cdot \frac{\partial}{\partial W}\right)=G_{A}{ }^{B} \frac{\partial}{\partial X^{B}}+W_{A}\left(X \cdot \frac{\partial}{\partial W}\right)
$$

we can clearly see that $X^{A} \nabla_{A}=0$, and moreover if the contracted tensor in (A.9) already satisfies the transverse condition, the action of the last term is trivial. We can express the action of $\nabla_{A}$ on such a tensor which is the representative of an $\operatorname{AdS}_{d+1}$ tensor as:

$$
\nabla_{B} T_{A_{1} \ldots A_{r}}(X)=G_{B}{ }^{C} G_{A_{1}}{ }^{C_{1}} \ldots G_{A_{r}}{ }^{C_{r}} \frac{\partial}{\partial X^{C}} T_{C_{1} \ldots C_{r}}(X) .
$$

In particular, it is worth noting that induced $\mathrm{AdS}_{d+1}$ metric $G_{A B}$ itself also satisfies transverse condition $X^{A} G_{A B}=G_{A B} X^{B}=0$, we have

$$
\nabla_{C} G_{A B}=G_{C} C^{\prime} G_{A} A^{\prime} G_{B}^{B^{\prime}} \frac{\partial}{\partial X^{C^{\prime}}} G_{A^{\prime} B^{\prime}}=0
$$

as required for $\nabla_{A}$ to be the metric covariant derivative in the embedding space. 


\section{B Integrals for three point geodesic Witten diagrams}

Scalar integral. Here we compute the integral associated with the three point scalar geodesic Witten diagram, which is frequently used in the main text:

$$
\mathcal{A}_{3}^{\Delta_{1} \Delta_{2} \Delta_{0}}\left(P_{1}, P_{2}, P_{0}\right) \equiv \int_{-\infty}^{\infty} d \lambda \frac{1}{\left(-2 P_{1} \cdot X(\lambda)\right)^{\Delta_{1}}} \frac{1}{\left(-2 P_{2} \cdot X(\lambda)\right)^{\Delta_{2}}} \frac{1}{\left(-2 P_{0} \cdot X(\lambda)\right)^{\Delta_{0}}} .
$$

where $X(\lambda)$ is given in (2.5). This can be computed readily using the integral definition of Beta function $\mathrm{B}(a, b)=\frac{\Gamma(a) \Gamma(b)}{\Gamma(a+b)}$, after the direct substitution of geodesic coordinate, we can express the integral above as:

$$
\begin{aligned}
& \mathcal{A}_{3}^{\Delta_{1} \Delta_{2} \Delta_{0}}\left(P_{1}, P_{2}, P_{0}\right)=P_{12}^{-\frac{1}{2}\left(\Delta_{1}+\Delta_{2}-\Delta_{0}\right)} P_{10}^{-\Delta_{0}} \int_{-\infty}^{\infty} d \lambda e^{\left(-\Delta_{1}+\Delta_{2}+\Delta_{0}\right) \lambda}\left(\frac{P_{20}}{P_{10}} e^{2 \lambda}+1\right)^{-\Delta_{0}} \\
& =P_{12}^{-\frac{1}{2}\left(\Delta_{1}+\Delta_{2}-\Delta_{0}\right)} P_{10}^{-\Delta_{0}}\left(\frac{P_{10}}{P_{20}}\right)^{\frac{1}{2}\left(-\Delta_{1}+\Delta_{2}+\Delta_{0}\right)} \int_{0}^{\infty} \frac{d \tilde{t}}{2 \tilde{t}} \tilde{t}^{\frac{1}{2}\left(-\Delta_{1}+\Delta_{2}+\Delta_{0}\right)}(\tilde{t}+1)^{-\Delta_{0}} \\
& =\frac{\beta_{\Delta_{12}, \Delta_{0}}}{P_{12}^{\frac{1}{2}\left(\Delta_{1}+\Delta_{2}-\Delta_{0}\right)} P_{20}^{\frac{1}{2}\left(\Delta_{2}+\Delta_{0}-\Delta_{1}\right)} P_{10}^{\frac{1}{2}\left(\Delta_{0}+\Delta_{1}-\Delta_{2}\right)}}
\end{aligned}
$$

where

$$
\beta_{\Delta_{12}, \Delta_{0}} \equiv \frac{1}{2} \mathrm{~B}\left(\frac{\Delta_{0}+\Delta_{12}}{2}, \frac{\Delta_{0}-\Delta_{12}}{2}\right)=\frac{\Gamma\left(\frac{\Delta_{0}+\Delta_{12}}{2}\right) \Gamma\left(\frac{\Delta_{0}-\Delta_{12}}{2}\right)}{2 \Gamma\left(\Delta_{0}\right)} .
$$

In the second line in (B.2), we have made the following change of integration variable: $\tilde{t}=\frac{P_{20}}{P_{10}} e^{2 \lambda}$.

Spin- $\boldsymbol{l}$ integral. Here we consider the spin- $l, l=0,1, \ldots J-1$ generalization of the computation for spin- $J$ case done in $(2.22)$. The corresponding three point interaction vertex is:

$$
g_{\Phi_{1} \Phi_{2} \Xi_{l}} \int_{X=X(\lambda)} d X \nabla^{C_{1}} \ldots \nabla^{C_{r}} \Phi_{1}(X) \nabla^{C_{r+1}} \ldots \nabla^{C_{J}} \Phi_{2}(X) \nabla_{C_{1}} \ldots \nabla_{C_{J-l}} \Xi(X)_{C_{J-l+1} \ldots C_{J}} .
$$

The vertex (B.4) generates the following three point geodesic Witten diagram:

$$
\begin{aligned}
& \int_{\gamma_{12}}(K \cdot \nabla)^{r} \frac{\mathcal{C}_{\Delta_{1}}}{\left(-2 P_{1} \cdot X\right)^{\Delta_{1}}}(K \cdot \nabla)^{J-r} \frac{\mathcal{C}_{\Delta_{2}}}{\left(-2 P_{2} \cdot X\right)^{\Delta_{2}}}(W \cdot \nabla)^{J-l}\left[\mathcal{C}_{h+i \nu, l} \frac{\left(2 X \cdot C_{0} \cdot W\right)^{l}}{\left(-2 P_{0} \cdot X\right)^{h+i \nu+l}}\right] \\
& =\mathcal{P}_{\Delta_{1}, \Delta_{2}, h+i \nu}^{l, J-r} \int_{\gamma_{12}} \frac{\left(2 P_{1} \cdot G \cdot K\right)^{r}\left(2 P_{2} \cdot G \cdot K\right)^{J-r}}{\left(-2 P_{1} \cdot X\right)^{\Delta_{1}+r}\left(-2 P_{2} \cdot X\right)^{\Delta_{2}+J-r}}\left[\frac{\left(2 P_{0} \cdot W\right)^{J-l}\left(2 X \cdot C_{0} \cdot W\right)^{l}}{\left(-2 P_{0} \cdot X\right)^{h+i \nu+l}}\right] \\
& =\mathcal{P}_{\Delta_{1}, \Delta_{2}, h+i \nu}^{l, J-r}(-1)^{J-r} \int_{-\infty}^{\infty} d \lambda \frac{\left(\frac{d X(\lambda)}{d \lambda} \cdot K\right)^{J}}{\left(-2 P_{1} \cdot X(\lambda)\right)^{\Delta_{1}}\left(-2 P_{2} \cdot X(\lambda)\right)^{\Delta_{2}}} \frac{\left(2 P_{0} \cdot W\right)^{J-l}\left(2 X(\lambda) \cdot C_{0} \cdot W\right)^{l}}{\left(-2 P_{0} \cdot X(\lambda)\right)^{h+i \nu+J}} \\
& =\mathcal{P}_{\Delta_{1}, \Delta_{2}, h+i \nu}^{l, J-r}(-1)^{J-r} J !\left(\frac{d-1}{2}\right)_{J} \int_{-\infty}^{\infty} d \lambda \frac{\left[2 \mathrm{~V}_{0,12}\right]^{l}\left(\frac{\left(-2 P_{0} \cdot P_{2}\right)}{\left(-2 P_{2} \cdot X(\lambda)\right)}-\frac{\left(-2 P_{0} \cdot P_{1}\right)}{\left(-2 P_{1} \cdot X(\lambda)\right)}\right)^{J-l}}{\left(-2 P_{1} \cdot X(\lambda)\right)^{\Delta_{1}}\left(-2 P_{2} \cdot X(\lambda)\right)^{\Delta_{2}}\left(-2 P_{0} \cdot X(\lambda)\right)^{h+i \nu+J}} \\
& =2^{l}(-1)^{J-r} J !\left(\frac{d-1}{2}\right)_{J} \mathbb{P}_{\Delta_{1}, \Delta_{2}, h+i \nu}^{l, J-r} \mathcal{C}_{h+i \nu, l} \beta_{\Delta_{12}, h+i \nu+l}\left[\begin{array}{ccc}
\Delta_{1} & \Delta_{2} & h+i \nu \\
0 & 0 & l \\
0 & 0 & 0
\end{array}\right]
\end{aligned}
$$


The overall factor is defined to be:

$$
\begin{aligned}
\mathcal{P}_{\Delta_{1}, \Delta_{2}, \frac{d}{2}+i \nu}^{l, J-r}= & \mathcal{C}_{\Delta_{1}} \mathcal{C}_{\Delta_{2}} \mathcal{C}_{h+i \nu, l}\left(\Delta_{1}\right)_{r}\left(\Delta_{2}\right)_{J-r}(h+i \nu+l)_{J-l}, \\
\mathbb{P}_{\Delta_{1}, \Delta_{2}, h+i \nu}^{l, J-r}= & \mathcal{C}_{\Delta_{1}} \mathcal{C}_{\Delta_{2}}\left(\Delta_{1}\right)_{r}\left(\Delta_{2}\right)_{J-r} \\
& \times \sum_{s=0}^{J-l} \frac{(J-l) !(-1)^{J-l-s}}{s !(J-l-s) !}\left(\frac{h+i \nu+l-\Delta_{12}}{2}\right)_{s}\left(\frac{h+i \nu+l+\Delta_{12}}{2}\right)_{J-l-s} .
\end{aligned}
$$

Up to an overall factor $\mathbb{P}_{\Delta_{1}, \Delta_{2}, h+i \nu}^{l,-r}$, which despite its dependence on $\nu$, does not introduce additional singularities for $\nu$ integration, we see that spin- $l$ case (B.5) takes exactly same expression for its spin- $J$ counterpart (2.22) with trivial substitution $J \rightarrow l$.

\section{Integrals for three point normal Witten diagrams}

Here we consider the integration of normal three point Witten diagram with scalar fields. The following calculation is based on [29]:

$$
I^{3-\mathrm{pt}} \equiv \int d X \frac{1}{\left(-2 P_{1} \cdot X\right)^{\Delta_{1}}} \frac{1}{\left(-2 P_{2} \cdot X\right)^{\Delta_{2}}} \frac{1}{\left(-2 P_{3} \cdot X\right)^{\Delta_{3}}} .
$$

Using the Schwinger parameterization,

$$
\frac{1}{\left(-P_{i} \cdot X\right)^{\Delta_{i}}}=\frac{1}{\Gamma\left(\Delta_{i}\right)} \int_{-\infty}^{\infty} \frac{d t_{i}}{t_{i}} t^{\Delta_{i}} e^{-\left(-2 P_{i} \cdot X\right) t_{i}}
$$

we can rewrite the integration as

$$
I^{3-\mathrm{pt}}=\frac{1}{\Gamma\left(\Delta_{1}\right) \Gamma\left(\Delta_{2}\right) \Gamma\left(\Delta_{2}\right)} \int_{-\infty}^{\infty} \frac{d t_{1}}{t_{1}} \frac{d t_{2}}{t_{2}} \frac{d t_{3}}{t_{3}} t_{1}^{\Delta_{1}} t_{2}^{\Delta_{2}} t_{3}^{\Delta_{3}} \int d X e^{2 Q \cdot X},
$$

where $Q$ is defined as $Q \equiv \sum_{i=1}^{3} t_{i} P_{i}$. Because $Q \cdot X$ is a scalar under the Lorentz transformation in the embedding space $\mathbb{M}^{d+1,1}$, we can choose $Q$ as $|Q|(1,1,0)$ where $|Q|^{2}=\sum_{i>j} t_{i} t_{j} P_{i j}$. Now the coordinate $X$ is parametrized as $\left(1, z^{2}+y^{2}, y^{\mu}\right) / z$, we can evaluate the AdS integration

$$
\begin{aligned}
\int d X e^{2 Q \cdot X} & =\int_{0}^{\infty} \frac{d z}{z} \int_{\mathbb{R}^{d}} \frac{d^{d} y}{z^{d}} e^{-\frac{|Q|}{z}\left(z^{2}+y^{2}+1\right)} \\
& =\pi^{h} \int_{0}^{\infty} \frac{d z}{z} \frac{1}{(z|Q|)^{h}} e^{-\frac{|Q|}{z}\left(z^{2}+1\right)} \\
& =\pi^{h} \int_{0}^{\infty} \frac{d z}{z} \frac{1}{z^{h}} e^{-\left(z+\frac{|Q|^{2}}{z}\right)}
\end{aligned}
$$

in the last line, $z$ is scaled as $z \rightarrow|Q| z$. Scaling $t_{i}$ as $t_{i} \rightarrow t_{i} \sqrt{z}$, we can perform the $z$ integration

$$
\begin{aligned}
I^{3-\mathrm{pt}} & =\frac{\pi^{h}}{\Gamma\left(\Delta_{1}\right) \Gamma\left(\Delta_{2}\right) \Gamma\left(\Delta_{2}\right)} \int_{-\infty}^{\infty} \frac{d t_{1}}{t_{1}} \frac{d t_{2}}{t_{2}} \frac{d t_{3}}{t_{3}} \int_{0}^{\infty} \frac{d z}{z} z^{\frac{\Delta_{1}+\Delta_{2}+\Delta_{3}-d}{2}} e^{-z-|Q|^{2}} \\
& =\frac{\pi^{h}}{\Gamma\left(\Delta_{1}\right) \Gamma\left(\Delta_{2}\right) \Gamma\left(\Delta_{2}\right)} \Gamma\left(\frac{\sum_{i=1}^{3} \Delta_{i}-d}{2}\right) \int_{-\infty}^{\infty} \frac{d t_{1}}{t_{1}} \frac{d t_{2}}{t_{2}} \frac{d t_{3}}{t_{3}} t_{1}^{\Delta_{1}} t_{2}^{\Delta_{2}} t_{3}^{\Delta_{3}} e^{-\sum_{i>j} t_{i} t_{j} P_{i j}}
\end{aligned}
$$


By utilizing the following parameterization:

$$
t_{1}=\sqrt{\frac{m_{1} m_{3}}{m_{2}}}, \quad t_{2}=\sqrt{\frac{m_{1} m_{2}}{m_{3}}}, \quad t_{3}=\sqrt{\frac{m_{2} m_{3}}{m_{1}}},
$$

the $t_{i}$ integration can be calculated as

$$
\begin{aligned}
\int_{-\infty}^{\infty} \frac{d t_{1}}{t_{1}} & \frac{d t_{2}}{t_{2}} \frac{d t_{3}}{t_{3}} t_{1}^{\Delta_{1}} t_{2}^{\Delta_{2}} t_{3}^{\Delta_{3}} e^{-\sum_{i>j} t_{i} t_{j} P_{i j}} \\
= & \frac{1}{2} \int_{0}^{\infty} \frac{d m_{1}}{m_{1}} \frac{d m_{2}}{m_{2}} \frac{d m_{3}}{m_{3}} m_{1}^{\frac{\Delta_{1}+\Delta_{2}-\Delta_{3}}{2}} m_{2}^{\frac{\Delta_{2}+\Delta_{3}-\Delta_{1}}{2}} m_{3}^{\frac{\Delta_{3}+\Delta_{1}-\Delta_{2}}{2}} e^{-m_{1} P_{12}-m_{2} P_{23}-m_{3} P_{31}} \\
= & \frac{1}{2} \Gamma\left(\frac{\Delta_{1}+\Delta_{2}-\Delta_{3}}{2}\right) \Gamma\left(\frac{\Delta_{1}-\Delta_{2}+\Delta_{3}}{2}\right) \Gamma\left(\frac{\Delta_{2}-\Delta_{1}+\Delta_{3}}{2}\right) \\
& \times P_{12}^{-\frac{1}{2}\left(\Delta_{1}+\Delta_{2}-\Delta_{3}\right)} P_{23}^{-\frac{1}{2}\left(\Delta_{2}+\Delta_{3}-\Delta_{1}\right)} P_{31}^{-\frac{1}{2}\left(\Delta_{3}+\Delta_{1}-\Delta_{2}\right)}
\end{aligned}
$$

Therefore the three point scalar diagram (C.1) can be evaluated as

$$
I^{3-\mathrm{pt}}=\mathcal{N}^{\Delta_{1}, \Delta_{2}, \Delta_{3}} \hat{\mathcal{A}}^{\Delta_{1}, \Delta_{2}, \Delta_{3}},
$$

where

$$
\begin{aligned}
& \mathcal{N}^{\Delta_{1}, \Delta_{2}, \Delta_{3}} \equiv \\
& \frac{\pi^{h} \Gamma\left(\frac{\sum_{i=1}^{3} \Delta_{i}-d}{2}\right)}{2 \Gamma\left(\Delta_{1}\right) \Gamma\left(\Delta_{2}\right) \Gamma\left(\Delta_{3}\right)} \Gamma\left(\frac{\Delta_{1}+\Delta_{2}-\Delta_{3}}{2}\right) \Gamma\left(\frac{\Delta_{1}-\Delta_{2}+\Delta_{3}}{2}\right) \Gamma\left(\frac{\Delta_{2}-\Delta_{1}+\Delta_{3}}{2}\right), \\
& \hat{\mathcal{A}}^{\Delta_{1}, \Delta_{2}, \Delta_{3}} \equiv P_{12}^{-\frac{1}{2}\left(\Delta_{1}+\Delta_{2}-\Delta_{3}\right)} P_{23}^{-\frac{1}{2}\left(\Delta_{2}+\Delta_{3}-\Delta_{1}\right)} P_{31}^{-\frac{1}{2}\left(\Delta_{3}+\Delta_{1}-\Delta_{2}\right)} .
\end{aligned}
$$

\section{Rewriting tensor structures and some useful identities}

In this appendix we consider more explicit proof of the statement that the three point geodesic Witten diagrams involving spins, formed by arbitrary Lorentz invariant vertices, can be expressed in terms of linear combination of box tensor basis, filling in some details for the general arguments given in [11].

Here we show that a transverse polynomial $\mathbb{Q}\left(P_{i}, Z_{i}\right)(i=1,2,3)$ be built only from $\mathrm{H}_{i j}$ and $\mathrm{V}_{i, j k}$. We assume that the polynomial $\mathbb{Q}\left(P_{i}, Z_{i}\right)$ has degree $l_{i}$ in $Z_{i}$ and it is transverse in each $Z_{i}$, in other wards, $\mathbb{Q}\left(P_{i}, Z_{i}\right)$ is invariant under the following shift of $Z_{i}$;

$$
Z_{i} \rightarrow Z_{i}+\alpha_{i} P_{i}
$$

where $\alpha_{i}$ are arbitrary constants. Because this polynomial $\mathbb{Q}$ do not have the Lorentz indices, $\mathbb{Q}$ can only consist of three scalar products; $P_{i} \cdot P_{j}, Z_{i} \cdot P_{j}$ and $Z_{i} \cdot Z_{j}$. The combination $Z_{i} \cdot Z_{j}$ to $\mathrm{H}_{i j}$ is replaced to $\mathrm{H}_{i j}$ and other scalar product through (3.4). Then $\mathbb{Q}$ can be represented as;

$$
\mathbb{Q}\left(P_{i}, Z_{i}\right)=\sum_{\left(m_{1}, m_{2}, m_{3}\right)=(0,0,0)}^{\left(l_{1}, l_{2}, l_{3}\right)} R_{m_{1}, m_{2}, m_{3}}\left(\left(P_{i} \cdot P_{j}\right),\left(Z_{i} \cdot P_{j}\right), \mathrm{H}_{i j}\right),
$$


where $R_{m_{1}, m_{2}, m_{3}}$ is a polynomial consisted of $P_{i} \cdot P_{j}, Z_{i} \cdot P_{j}$ and $\mathrm{H}_{i j}$ and it contains $m_{i} Z_{i}$ besides $\mathrm{H}_{i j}$. We can decompose $R_{m_{1}, m_{2}, m_{3}}$ further;

$$
R_{m_{1}, m_{2}, m_{3}}=\sum_{n=0}^{m_{1}} c_{n, m_{1}-n}\left(Z_{1} \cdot P_{2}\right)^{n}\left(Z_{1} \cdot P_{3}\right)^{m_{1}-n} .
$$

Here we focus on the specific $Z_{1}$ dependence. The coefficient $c_{n, m_{1}-n}$ depends on $Z_{1}$ only through $\mathrm{H}_{12}$ or $\mathrm{H}_{31}$. $\mathbb{Q}$ should satisfy the transverse condition;

$$
\mathbb{Q}\left(P_{i}, Z_{i}\right)=\mathbb{Q}\left(P_{i}, Z_{i}+\beta_{i} P_{i}\right),
$$

therefore the following equation should be satisfied

$$
\frac{\partial}{\partial \beta}\left[\sum_{n=0}^{m_{1}} c_{n, m_{1}-n}\left(Z_{1} \cdot P_{2}+\beta P_{1} \cdot P_{2}\right)^{n}\left(Z_{1} \cdot P_{3}+\beta P_{1} \cdot P_{3}\right)^{m_{1}-n}\right]=0 .
$$

Because this condition should satisfied at each order of $\left(Z_{1} \cdot P_{2}\right)$ or $\left(Z_{1} \cdot P_{2}\right)$, we can obtain the following recursion equation;

$$
\left(m_{1}-n\right) c_{n, m_{1}-n}\left(P_{1} \cdot P_{3}\right)+(n+1) c_{n+1, m_{1}-n-1}\left(P_{1} \cdot P_{2}\right)=0 .
$$

According to this relation, $c_{n, m_{1}-n}$ is determined as

$$
c_{n, m_{1}-n}={ }_{m_{1}} C_{n}\left(-\frac{P_{1} \cdot P_{3}}{P_{1} \cdot P_{2}}\right)^{n} c_{0, m_{1}} .
$$

Then the decomposition in (D.3) is just a binomial expansion and $R_{m_{1}, m_{2}, m_{3}}$ can be rewritten as;

$$
R_{m_{1}, m_{2}, m_{3}}=c_{0, m_{1}}\left(-\frac{P_{2} \cdot P_{3}}{P_{1} \cdot P_{2}} \mathrm{~V}_{1,23}\right)^{m_{1}}
$$

The discussions for $Z_{2}$ and $Z_{3}$ go through similarly. Therefore transverse polynomials $\mathbb{Q}$ should depend on depends on $Z_{i}$ only through $\mathrm{H}_{i j}$ and $\mathrm{V}_{i, j k}$.

Here we also list out few useful identities which involve the contractions among triplets of anti-symmetric $\mathrm{C}_{i}^{A B}$ associated with $\mathcal{O}_{\Delta_{i}, l_{i}}\left(P_{i}, Z_{i}\right), i=1,2,3$, which are useful in the actual explicit computations:

$$
\begin{aligned}
\left(\mathrm{C}_{i} \cdot \mathrm{C}_{j}\right)_{A B}= & \frac{1}{2} \frac{\mathrm{H}_{i j}}{\left(P_{i} \cdot P_{j}\right)} P_{i A} P_{j B}-\frac{\left(P_{j} \cdot \mathrm{C}_{i}\right)_{A}\left(P_{i} \cdot \mathrm{C}_{j}\right)_{B}}{\left(P_{i} \cdot P_{j}\right)} \\
\left(\mathrm{C}_{i} \cdot \mathrm{C}_{j} \cdot \mathrm{C}_{k}\right)_{A C}= & \frac{\mathrm{H}_{i j}}{2} \frac{P_{i A}\left(P_{j} \cdot \mathrm{C}_{k}\right)_{C}}{\left(P_{i} \cdot P_{j}\right)}-\frac{\mathrm{H}_{j k}}{2} \frac{P_{k C}\left(P_{j} \cdot \mathrm{C}_{i}\right)_{A}}{\left(P_{i} \cdot P_{j}\right)} \\
& +\frac{\left(P_{j} \cdot \mathrm{C}_{i}\right)_{A}\left(P_{j} \cdot \mathrm{C}_{k}\right)_{C}}{\left(P_{i} \cdot P_{j}\right)\left(P_{j} \cdot P_{k}\right)}\left(P_{k} \cdot \mathrm{C}_{j} \cdot P_{i}\right) \\
\left(\mathrm{C}_{i} \cdot \mathrm{C}_{j} \cdot \mathrm{C}_{i}\right)_{A C}= & -\frac{\mathrm{H}_{i j}}{2} \mathrm{C}_{i A C} \\
\left(\mathrm{C}_{i} \cdot \mathrm{C}_{j} \cdot \mathrm{C}_{k} \cdot \mathrm{C}_{j}\right)_{A D}= & \frac{-\mathrm{H}_{i j} \mathrm{H}_{j k}}{4} \frac{P_{i A} P_{j D}}{\left(P_{i} \cdot P_{j}\right)}+\frac{\mathrm{H}_{j k}\left(P_{j} \cdot \mathrm{C}_{i}\right)_{A}\left(P_{i} \cdot \mathrm{C}_{j}\right)_{D}}{2\left(P_{i} \cdot P_{j}\right)}
\end{aligned}
$$




$$
\begin{aligned}
\left(\mathrm{C}_{i} \cdot \mathrm{C}_{j} \cdot \mathrm{C}_{k} \cdot \mathrm{C}_{i}\right)_{A D}= & \frac{\mathrm{H}_{i j} \mathrm{H}_{k i}}{4} \frac{\left(P_{j} \cdot P_{k}\right) P_{i A} P_{i D}}{\left(P_{i} \cdot P_{j}\right)\left(P_{k} \cdot P_{i}\right)}-\frac{1}{4} \mathrm{~V}_{j, k i} \mathrm{~V}_{k, i j}\left(P_{j} \cdot \mathrm{C}_{i}\right)_{A}\left(P_{j} \cdot \mathrm{C}_{i}\right)_{D} \\
& -\frac{1}{2} \mathrm{H}_{j k} \frac{\left(P_{j} \cdot \mathrm{C}_{i}\right)_{A}\left(P_{k} \cdot \mathrm{C}_{j}\right)_{D}}{\left(P_{i} \cdot P_{j}\right)}-\frac{1}{2} \mathrm{H}_{i j} \frac{\mathrm{V}_{k, i j} P_{\mathrm{iA}}\left(P_{k} \cdot \mathrm{C}_{i}\right)_{D}}{\left(P_{k} \cdot P_{i}\right)} \\
& +\frac{1}{2} \mathrm{H}_{k i} \frac{\mathrm{V}_{j, k i}\left(P_{j} \cdot P_{k}\right)}{\left(P_{i} \cdot P_{j}\right)}\left(P_{j} \cdot \mathrm{C}_{i}\right)_{A} P_{i D} .
\end{aligned}
$$

Along with the obvious identity $\left(\mathrm{C}_{i} \cdot \mathrm{C}_{i}\right)_{A B}=0$, all other successive contractions of $\mathrm{C}_{i}^{A B}$ can obtained by repeatedly using these identities. It should be clear from above that any invariant scalars constructed from contacting (D.9)-(D.13) with either a pair of $P_{i}$ or a $\mathrm{C}_{i A B}$ are all transverse polynomials and can all be expressed in terms products of $\mathrm{H}_{i j}$ and $\mathrm{V}_{i, j k}$ with coefficients only depending on $\left(P_{i} \cdot P_{j}\right)$. These will be needed when we study the tensor structures of the three point geodesic Witten diagrams.

\section{E Computational details for decomposition analysis}

In section 5, we have demonstrated that ordinary four point scalar Witten diagram can be written as a summation of four point scalar CPWs. In this case, each CPW is proportional to a GWD, this leads us to the claimed results, here we present the computational details to see such decomposition. In (5.10), we can replace $\hat{\mathcal{A}}$ with a geodesic diagram as follows;

$$
\begin{aligned}
I_{(J, l)}^{\Delta_{1}, \Delta_{2}, h+i \nu}= & \sum_{p=0}^{J-l}{ }_{J-l} C_{p} \frac{(-2)^{l}\left(\Delta_{2}\right)_{J}(h+i \nu+l)_{J-l}}{(h+i \nu+J-l-p)_{l}}\left(-2 P_{20}\right)^{J-l-p} \mathcal{N}^{\Delta_{1}, \Delta_{2}+J-p, h+i \nu+J-l-p} \\
& \times\left(\mathrm{D}_{02}\right)^{l} \hat{\mathcal{A}}^{\Delta_{1}, \Delta_{2}+J-p, h+i \nu+J-l-p} \\
= & \sum_{p=0}^{J-l} J-l C_{p} \frac{\left(\Delta_{2}\right)_{J}(h+i \nu+l)_{J-l}}{(h+i \nu+J-l-p)_{l}}(-2)^{J-p} \mathcal{N}^{\Delta_{1}, \Delta_{2}+J-p, h+i \nu+J-l-p} \\
& \times\left(\frac{\Delta_{12}+h+i \nu-l}{2}\right)_{l}\left(-V_{0,12}\right)^{l} \mathcal{A}^{\Delta_{1}, \Delta_{2}, h+i \nu+l} \\
= & \sum_{p=0}^{J-l} \tilde{\mathcal{R}}_{\Delta_{1}, \Delta_{2}, h+i \nu}^{J l, p}\left(\frac{\Delta_{1}+\Delta_{2}+l-h \pm i \nu}{2}\right) \\
& \times \frac{1}{l !\left(\frac{d-1}{2}\right)_{l}} \int_{\gamma_{12}} d \lambda \frac{1}{\left(-2 P_{1} \cdot X\right)^{\Delta_{1}}}(K \cdot \nabla)^{l} \frac{1}{\left(-2 P_{2} \cdot X\right)^{\Delta_{2}}} \frac{\left(W \cdot V_{0}(X)\right)^{l}}{\left(-2 P_{0} \cdot X\right)^{h+i \nu+l}},
\end{aligned}
$$

where in the last line we used the following relation:

$$
\begin{aligned}
& \frac{1}{l !\left(\frac{d-1}{2}\right)_{l}} \int_{\gamma_{12}} d \lambda \frac{1}{\left(-2 P_{1} \cdot X\right)^{\Delta_{1}}}(K \cdot \nabla)^{l} \frac{1}{\left(-2 P_{2} \cdot X\right)^{\Delta_{2}}} \frac{\left(W \cdot V_{0}(X)\right)^{l}}{\left(-2 P_{0} \cdot X\right)^{h+i \nu+l}} \\
& =\left(\Delta_{2}\right)_{l}(-2)^{l} \beta^{\Delta_{1}, \Delta_{2}, h+i \nu+l}\left(-V_{0,12}\right)^{l} \mathcal{A}^{\Delta_{1}, \Delta_{2}, h+i \nu+l}
\end{aligned}
$$

and $\tilde{\mathcal{R}}_{\Delta_{1}, \Delta_{2}, h+i \nu}^{J, l, p}$ is a regular function of $\nu$ :

$$
\begin{aligned}
\tilde{\mathcal{R}}_{\Delta_{1}, \Delta_{2}, h+i \nu}^{J, l, p} \equiv & { }_{J-l} C_{p} \frac{\pi^{h}(-2)^{J-l-p}\left(\Delta_{2}+l\right)_{J-l}}{\Gamma\left(\Delta_{1}\right) \Gamma\left(\Delta_{2}+J-p\right)}(h+i \nu+J-p)_{p} \\
& \times\left(\frac{-\Delta_{12}+h+i \nu+l}{2}\right)_{J-l-p}\left(\frac{\Delta_{1}+\Delta_{2}-h+i \nu+l}{2}\right)_{J-l-p} .
\end{aligned}
$$


Using (E.1), now we can rewrite (5.13) as:

$$
\begin{aligned}
W_{(0,0), J,(0,0)}^{4-\text { t. }}= & \sum_{l=0}^{J} \int_{\partial} d P_{0} \int_{-\infty}^{\infty} d \nu a_{l}(\nu) \frac{\nu^{2}}{\pi l !(h-1) !} \sum_{p=0}^{J-l} \sum_{p^{\prime}=0}^{J-l} \tilde{\mathcal{R}}_{\Delta_{1}, \Delta_{2}, h+i \nu}^{J, l, p} \tilde{\mathcal{R}}_{\Delta_{3}, \Delta_{4}, h-i \nu}^{J, l, p^{\prime}} \quad \text { (E.4) } \\
& \times \Gamma\left(\frac{\Delta_{1}+\Delta_{2}+l-h \pm i \nu}{2}\right) \Gamma\left(\frac{\Delta_{3}+\Delta_{4}+l-h \pm i \nu}{2}\right) \\
& \times \frac{\mathcal{C}_{h+i \nu, l}}{l !\left(\frac{d-1}{2}\right)_{l}} \int_{\gamma_{12}} d \lambda \frac{1}{\left(-2 P_{1} \cdot X\right)^{\Delta_{1}}}(K \cdot \nabla)^{l} \frac{1}{\left(-2 P_{2} \cdot X\right)^{\Delta_{2}}} \frac{\left(W \cdot V_{0}\left(X, D_{Z_{0}}\right)\right)^{l}}{\left(-2 P_{0} \cdot X\right)^{h+i \nu+l}} \\
& \times \frac{\mathcal{C}_{h-i \nu, l}}{l !\left(\frac{d-1}{2}\right)_{l}} \int_{\gamma_{34}} d \lambda^{\prime} \frac{1}{\left(-2 P_{3} \cdot \tilde{X}\right)^{\Delta_{3}}}(\tilde{K} \cdot \tilde{\nabla})^{l} \frac{1}{\left(-2 P_{4} \cdot \tilde{X}\right)^{\Delta_{4}}} \frac{\left(\tilde{W} \cdot V_{0}\left(\tilde{X}, Z_{0}\right)\right)^{l}}{\left(-2 P_{0} \cdot \tilde{X}\right)^{h-i \nu+l}} .
\end{aligned}
$$

From (2.13) and (2.15), the two bulk to boundary propagator can be glued together:

$$
\begin{gathered}
\int_{\partial} d P_{0} a_{l}(\nu) \frac{\nu^{2}}{\pi l !(h-1) !} \mathcal{C}_{h+i \nu, l} \frac{\left(W \cdot V_{0}\left(X, D_{Z_{0}}\right)\right)^{l}}{\left(-2 P_{0} \cdot X\right)^{h+i \nu+l}} \mathcal{C}_{h-i \nu, l} \frac{\left(\tilde{W} \cdot V_{0}\left(\tilde{X}, Z_{0}\right)\right)^{l}}{\left(-2 P_{0} \cdot \tilde{X}\right)^{h-i \nu+l}} \\
=\frac{i \nu}{2 \pi}\left(\Pi_{h+i \nu, l}(X, \tilde{X} ; W, \tilde{W})-\Pi_{h-i \nu, l}(X, \tilde{X} ; W, \tilde{W})\right)
\end{gathered}
$$

then (E.4) becomes

$$
\begin{aligned}
W_{(0,0), J,(0,0)}^{4-\mathrm{pt.}}= & \sum_{l=0}^{J} \int_{-\infty}^{\infty} d \nu a_{l}(\nu) \frac{i \nu}{2 \pi} \sum_{p=0}^{J-l} \sum_{p^{\prime}=0}^{J-l} \tilde{\mathcal{R}}_{\Delta_{1}, \Delta_{2}, h+i \nu}^{J, l, p} \tilde{\mathcal{R}}_{\Delta_{3}, \Delta_{4}, h-i \nu}^{J, l, p^{\prime}} \\
& \times \Gamma\left(\frac{\Delta_{1}+\Delta_{2}+l-h \pm i \nu}{2}\right) \Gamma\left(\frac{\Delta_{3}+\Delta_{4}+l-h \pm i \nu}{2}\right) \\
& \times\left(\mathcal{W}_{h+i \nu, l}\left(P_{i}\right)-\mathcal{W}_{h-i \nu, l}\left(P_{i}\right)\right)
\end{aligned}
$$

where $\mathcal{W}_{\Delta, l}\left(P_{i}\right)$ is a four point GWD;

$$
\begin{aligned}
\mathcal{W}_{\Delta, l}\left(P_{i}\right)= & \frac{1}{\left(l !\left(\frac{d-1}{2}\right)_{l}\right)^{2}} \int_{\gamma_{12}} d \lambda \int_{\gamma_{34}} d \lambda^{\prime} \frac{1}{\left(-2 P_{1} \cdot X\right)^{\Delta_{1}}}(K \cdot \nabla)^{l} \frac{1}{\left(-2 P_{2} \cdot X\right)^{\Delta_{2}}} \\
& \times \frac{1}{\left(-2 P_{3} \cdot \tilde{X}\right)^{\Delta_{3}}}(\tilde{K} \cdot \tilde{\nabla})^{l} \frac{1}{\left(-2 P_{4} \cdot \tilde{X}\right)^{\Delta_{4}}} \\
& \times\left(\Pi_{h+i \nu, l}(X, \tilde{X} ; W, \tilde{W})-\Pi_{h-i \nu, l}(X, \tilde{X} ; W, \tilde{W})\right) .
\end{aligned}
$$

In (E.6), the intermediate states are determined by the pole structure of $\nu$ integration. The $\nu$ dependence is similar as in (5.17), the single trace contribution comes form the highest spin coefficient $a_{J}(\nu)$, and the double trace ones come from the gamma functions. In this more direct computation, we can see the decomposition into GWDs without passing through CPWs.

Open Access. This article is distributed under the terms of the Creative Commons Attribution License (CC-BY 4.0), which permits any use, distribution and reproduction in any medium, provided the original author(s) and source are credited. 


\section{References}

[1] E. Hijano, P. Kraus, E. Perlmutter and R. Snively, Witten Diagrams Revisited: The AdS Geometry of Conformal Blocks, JHEP 01 (2016) 146 [arXiv:1508.00501] [INSPIRE].

[2] D.Z. Freedman, S.D. Mathur, A. Matusis and L. Rastelli, Correlation functions in the CFT(d)/AdS(d+1) correspondence, Nucl. Phys. B 546 (1999) 96 [hep-th/9804058] [INSPIRE].

[3] E. D'Hoker, D.Z. Freedman, S.D. Mathur, A. Matusis and L. Rastelli, Graviton exchange and complete four point functions in the AdS/CFT correspondence, Nucl. Phys. B 562 (1999) 353 [hep-th/9903196] [inSPIRE].

[4] C. Sleight and M. Taronna, Spinning Witten Diagrams, arXiv:1702.08619 [InSPIRE].

[5] E. D'Hoker and D.Z. Freedman, Supersymmetric gauge theories and the AdS/CFT correspondence, hep-th/0201253 [INSPIRE].

[6] S. Rychkov, EPFL Lectures on Conformal Field Theory in D $\geq 3$ Dimensions, arXiv: 1601.05000.

[7] D. Simmons-Duffin, The Conformal Bootstrap, arXiv:1602.07982 [INSPIRE].

[8] F.A. Dolan and H. Osborn, Conformal Partial Waves: Further Mathematical Results, arXiv:1108.6194 [INSPIRE].

[9] D. Simmons-Duffin, Projectors, Shadows and Conformal Blocks, JHEP 04 (2014) 146 [arXiv: 1204.3894] [INSPIRE].

[10] M.S. Costa, J. Penedones, D. Poland and S. Rychkov, Spinning Conformal Blocks, JHEP 11 (2011) 154 [arXiv:1109.6321] [INSPIRE].

[11] M.S. Costa, J. Penedones, D. Poland and S. Rychkov, Spinning Conformal Correlators, JHEP 11 (2011) 071 [arXiv:1107.3554] [INSPIRE].

[12] E. Joung and M. Taronna, Cubic interactions of massless higher spins in (A)dS: metric-like approach, Nucl. Phys. B 861 (2012) 145 [arXiv:1110.5918] [INSPIRE].

[13] C. Sleight and M. Taronna, Higher Spin Interactions from Conformal Field Theory: The Complete Cubic Couplings, Phys. Rev. Lett. 116 (2016) 181602 [arXiv:1603.00022] [INSPIRE].

[14] M.S. Costa, V. Gonçalves and J. Penedones, Spinning AdS Propagators, JHEP 09 (2014) 064 [arXiv: 1404.5625] [INSPIRE].

[15] X. Bekaert, J. Erdmenger, D. Ponomarev and C. Sleight, Towards holographic higher-spin interactions: Four-point functions and higher-spin exchange, JHEP 03 (2015) 170 [arXiv: 1412.0016] [INSPIRE].

[16] A. Castro, E. Llabrés and F. Rejon-Barrera, Geodesic Diagrams, Gravitational Interactions \& OPE Structures, arXiv:1702.06128 [INSPIRE].

[17] E. Dyer, D.Z. Freedman and J. Sully, Spinning Geodesic Witten Diagrams, arXiv:1702.06139 [INSPIRE].

[18] M. Nishida and K. Tamaoka, Geodesic Witten diagrams with an external spinning field, arXiv:1609.04563 [INSPIRE].

[19] B. Czech, L. Lamprou, S. McCandlish, B. Mosk and J. Sully, A Stereoscopic Look into the Bulk, JHEP 07 (2016) 129 [arXiv:1604.03110] [INSPIRE]. 
[20] F.A. Dolan and H. Osborn, Conformal partial waves and the operator product expansion, Nucl. Phys. B 678 (2004) 491 [hep-th/0309180] [INSPIRE].

[21] M. Isachenkov and V. Schomerus, Superintegrability of d-dimensional Conformal Blocks, Phys. Rev. Lett. 117 (2016) 071602 [arXiv: 1602.01858] [INSPIRE].

[22] H.-Y. Chen and J.D. Qualls, Quantum Integrable Systems from Conformal Blocks, arXiv: 1605.05105 [INSPIRE].

[23] X. Bekaert, J. Erdmenger, D. Ponomarev and C. Sleight, Quartic AdS Interactions in Higher-Spin Gravity from Conformal Field Theory, JHEP 11 (2015) 149 [arXiv: 1508.04292] [INSPIRE].

[24] M.S. Costa and T. Hansen, Conformal correlators of mixed-symmetry tensors, JHEP 02 (2015) 151 [arXiv:1411.7351] [INSPIRE].

[25] M.S. Costa, T. Hansen, J. Penedones and E. Trevisani, Projectors and seed conformal blocks for traceless mixed-symmetry tensors, JHEP 07 (2016) 018 [arXiv: 1603.05551] [INSPIRE].

[26] M.S. Costa, T. Hansen, J. Penedones and E. Trevisani, Radial expansion for spinning conformal blocks, JHEP 07 (2016) 057 [arXiv: 1603.05552] [INSPIRE].

[27] C. Sleight, Interactions in Higher-Spin Gravity: a Holographic Perspective, arXiv: 1610.01318 [INSPIRE].

[28] M.S. Costa, V. Goncalves and J. Penedones, Conformal Regge theory, JHEP 12 (2012) 091 [arXiv: 1209.4355] [INSPIRE].

[29] J. Penedones, Writing CFT correlation functions as AdS scattering amplitudes, JHEP 03 (2011) 025 [arXiv: 1011.1485] [INSPIRE].

[30] V. Gonçalves, J. Penedones and E. Trevisani, Factorization of Mellin amplitudes, JHEP 10 (2015) 040 [arXiv: 1410.4185] [INSPIRE]. 